\title{
ANÁLISIS COMPARATIVO DE DESECHOS DE TALLA EN CONTEXTOS DEL PLEISTOCENO FINAL/HOLOCENO TEMPRANO DE CHILE Y ARGENTINA
}

\author{
NORA FLEGENHEIMER* \& ROXANA CATTÁNEO**
}

\section{RESUMEN}

Se presenta el análisis comparativo de conjuntos de desechos de talla lítica provenientes de sitios tempranos de la región pampeana argentina y de la Patagonia chilena asociados a puntas de proyectil tipo Fell 1 o cola de pescado. El objetivo es avanzar en la discusión sobre las formas de organizarse y sobre la vinculación entre prácticas sociales y lugares en el pasado. Para ello se aplica la metodología de análisis nodular mínimo (Larson \& Kornfeld 1997) y el análisis no tipológico que se basa en modelos de extracción de lascas (Ingbar et al. 1989). Los resultados se discuten en relación a aquéllos ya obtenidos aplicando otra metodología en el caso pampeano y se los consideran una exploración útil del caso patagónico.

En la visión comparativa de los resultados resalta la discontinuidad de las trayectorias en todos los conjuntos, ya que prácticamente sólo un segmento de la secuencia de manufactura está representado en cada sitio. También resalta la semejanza en las curvas obtenidas para Cueva Fell, Cerro El Sombrero Abrigo 1 y Cima. Se plantea que, las actividades de talla sobre distintas materias primas desarrolladas en cada sitio parecen estar en gran medida ligadas a las prácticas vinculadas a ese espacio en sí, y no sólo a la trayectoria esperable de la roca en relación a su calidad, disponibilidad y proveniencia. Finalmente, resulta interesante que esta modalidad sea compartida en regiones tan distantes como Pampa y el extremo sur de Patagonia por gente que también compartió los conocimientos necesarios para fabricar instrumentos como colas de pescado y piedras discoidales. Por ello, a partir de este trabajo proponemos que además de los conocimientos prácticos de cómo realizar una tarea posiblemente se estuvieran compartiendo también otras cuestiones más relacionadas a la esfera de los vínculos entre la gente y su entorno, o sea, aquellas que conciernen a la compleja trama de decisiones que implica la tecnología entendida en su sentido amplio.

PALABRAS CLAVE: desechos líticos, poblamiento temprano, tecnología, Cono Sur.

* CONICET-Área de Arqueología y Antropología. Municipalidad de Necochea. Buenos Aires, Argentina. Calle 49 No.1655. (7630) Necochea. noraf@necocheanet.com.ar.

*: Instituto de Antropología de Córdoba. Laboratorio de Análisis Macro y Microscópico de Materiales Líticos. IDACOR-CONICETMuseo de Antropología. Facultad de Filosofía y Humanidades. Universidad Nacional de Córdoba.- Av. Irigoyen 174 (5000) Córdoba, Argentina. roxanacattaneo@gmail.com. 


\section{COMPARATIVE FLAKE ANALYSIS IN LATE PLEISTOCEN EARLY HOLOCENE CONTEXTS OF CHILE AND ARGENTINA}

\section{ABSTRACT}

A comparative study of flakes associated to Fell 1 or fishtail projectile points from early sites in the Argentinian Pampa and the Chilean Patagonia is presented. The aim is to advance in the discussion regarding the different attitudes of early settlers and the link between social practices and landscape in the past. Minimum nodular analysis and non typological analysis were applied. Results obtained through these methodologies are discussed in relation to those previously obtained for the Pampean case and are considered a useful explorative study for the Patagonian sample.

When results are compared, the discontinuity in the representation of flaking trajectories is remarkable; each site exhibits only a segment of the manufacturing sequence. There is also a similarity between the curves obtained at Fell's Cave, Cerro El Sombrero Abrigo 1 and Cima. It is proposed that flaking activities on different raw materials are therefore in some cases more associated to place related practices than to raw material characteristics. Finally, it is interesting to point out that people who lived far away probably shared both practical knowledge about fishtail projectile point and discoidal stone manufacturing and the way in which social activities are carried out. This last issue includes aspects related to people and their relationship with their landscape, as well as those concerning the complex network of decisions that technology involves.

KEY WORDS: flakes, early peopling, technology, Southern Cone.

\section{INTRODUCCIÓN}

Nuestro objetivo en este trabajo es presentar los resultados de un estudio cuantitativo, exploratorio y comparativo de desechos de talla de sitios contemporáneos y distantes entre sí. Estudiamos conjuntos de desechos de talla lítica de distintas colecciones de sitios tempranos de la región pampeana argentina y de la Patagonia chilena a fin de entender de qué modo se dieron los procesos de manufactura del instrumental asociado a la producción de puntas de proyectil tipo cola de pescado o Fell 1 (Bird 1938; 1988). A través de este enfoque buscamos mejorar nuestras interpretaciones sobre cómo la gente organizó sus actividades y sobre la vinculación entre prácticas sociales y lugares en el pasado. En los últimos años, algunos de estos temas han sido tratados por otros autores desde varios enfoques tanto para la región de Magallanes (Prieto 1997; Massone \& Prieto 2004; Jackson 2007; Borrero \& Charlin 2010) como para la pampeana (Mazzanti 2003; Martínez 2006; ver síntesis en Politis 2008).

Desde distintas perspectivas metodológicas se considera que los desechos de talla, abundantes y muy frecuentes en el registro arqueológico, son adecuados para comprender diversos aspectos de la tecnología lítica. El enfoque particular con el que hemos estudiado dichos conjuntos aquí son el análisis Nodular (Larson \& Kornfeld 191997) y el análisis no tipológico (Ingbar et al. 1989). Ambos requieren una introducción para entender el porqué de la propuesta, dado que no son de aplicación frecuente en el cono sur. Por otro lado, los productos de la reducción lítica y los distintos métodos empleados para su estudio han recibido considerable atención en la literatura internacional de los últimos 30 años.

Así, los desechos han sido un tema central de discusión desde los trabajos clásicos de Sullivan \& Rozen (1985) y Rozen \& Sullivan (1989) donde eran usados para medir los efectos de la reducción de núcleos vs. la producción de instrumentos, hasta los de sus continuadores (Teltser 1991; Kuijt \& Rusell 1993 y para una revisión histórica crítica consultar Shott 1994; Prentiss 1998; Bamforth \& Finlay 2008). En Argentina, los procedimientos aplicados a su sistematización se han basado tanto en categorías tecnológicas o morfológicas, como en una combinación de ambas (Aschero 1975, 1983; Orquera y Piana 1987; Bellelli et al. 1985-87; Nami 1991; Espinosa 1998; Martínez et al. 1997-98; Cattáneo 2002, por mencionar algunos casos).

En tal sentido, a partir de los desechos de 
talla no sólo se pueden hacer interpretaciones sobre el proceso de manufactura sino también sobre las actividades desarrolladas en los diversos espacios y sobre la existencia de actores sociales. Por ello consideramos que aún existen varios aspectos interesantes por explorar en torno a los mismos y algunos ejemplos de ello son su utilidad para abordar temas tales como las propiedades de las materias primas (Bradbury \& Franklin 2000; Ashton \& White 2003) o las historias de vida de los objetos (Boeda 1996; Audouze 1999; Andrefsky 2005) y cómo estos aspectos se vinculan en interpretaciones más completas sobre los conjuntos líticos (Dibble 1997; Ashton \& White 2003).

Dentro de este contexto, el análisis nodular y el análisis no tipológico se han aplicado conjuntamente a algunos casos de estudio del cono sur (Cattáneo 2002, 2006, Sario 2007; 2009; Charlin 2009; Sario \& Pautassi 2010); para casos de Norteamérica ver por ejemplo Frison (1974) o Kelly (1985). Esta metodología en Argentina hasta el momento se empleó en dos ámbitos: en conjuntos líticos de grupos cazadores-recolectores de la Patagonia meridional, donde se buscó establecer la relación entre el uso de las fuentes de aprovisionamiento y la funcionalidad de los asentamientos (Cattáneo 2002, 2006, Charlin 2009) y en distintas localidades de la región central del país, en Córdoba y San Luis (Sario 2007, 2009; Pautassi y Sario 2011, Sario \& Pautassi 2012).

Por otro lado, durante varios años se estudiaron conjuntos líticos que provienen de distintos sitios de la región pampeana correspondientes a la transición Pleistoceno-Holoceno (Flegenheimer 1986/1987, 1988, 1991, 2004) empleando la tipología propuesta por Aschero (1975, 1983). Aunque los desechos fueron analizados con otra metodología, los objetivos de estudio se superpusieron parcialmente con los del sur del país, es decir, en ambas regiones se buscó correlacionar los artefactos y las fuentes de materias primas y establecer las características de las actividades desarrolladas en los sitios.

En este trabajo analizamos algunos conjuntos de desechos de talla asociados a la producción de instrumentos que incluyen puntas de proyectil cola de pescado o Fell 1 (Bird 1938, 1988). Se eligieron tres localidades donde se recuperaron puntas de este tipo; estas presentan un amplio rango de variabilidad en cuanto a sus tamaños, procesos de manufactura y morfología (Flegenheimer 1999) (Fig. 1). Las puntas en sí han sido analizadas a partir de distintas perspectivas (p.e., Mayer Oakes 1973; Politis1991; Nami 1993-94, 2003; Gnecco 2000; Castiñeira et al. 2011) e inclusive, su producción ha sido estudiada en detalle a través de un proyecto experimental por H. Nami (2003).

La muestra analizada (Fig. 2) corresponde a tres sitios de la región Pampeana argentina: Cima y Abrigo 1 de la localidad Cerro El Sombrero, y el Sitio 1 de la localidad Cerro La China (Flegenheimer 1986/1987, 2003, 2004). La muestra también incluye un sitio de la región patagónica chilena: Cueva Fell, colección del American Museum of Natural History (Nueva York, EE.UU.) y colección del Instituto de la Patagonia (Punta Arenas, Chile) (Massone 1981, 1996; Nami 1997, 1999, 2003; Schidlowsky 2001; Jackson \& Prieto 2005; Jackson 2007; Charlin 2009; Borrero \& Charlin 2010).

Nuestro fin último es analizar cómo se ha dado el proceso de manufactura en cada espacio y evaluar las modalidades reconocidas para el caso patagónico frente al pampeano. Asimismo, el trabajo tiene un fin metodológico ya que, como mencionamos, algunos de los desechos fueron ya analizados con otra metodología (Flegenheimer 1988, 1991), lo cual permitirá comparar sus potenciales interpretativos. También con este trabajo exploramos la idea de que la producción lítica al igual que la tecnología en general involucra una red de decisiones complejas. Éstas, entraman tanto aquéllas de orden inmediato y material, ente ellas, las que resuelven las constricciones que impone la materia prima y su distribución en el paisaje, así como también aquéllas de orden social, más intangibles, como los conocimientos y prácticas compartidas. Es dentro de este marco que nos parece interesante considerar las diferencias y en especial, las recurrencias entre los procesos de producción en estas dos regiones tan distantes.

\section{EL ENFOQUE METODOLÓGICO}

En este trabajo hemos utilizado las herramientas mencionadas para separar conjuntos de desechos de una materia prima y luego hemos proseguido a aplicar el análisis no tipológico. En este terreno, durante los últimos 25 años se desarrollaron estudios experimentales en desechos de talla (ver citas previas). A partir de los mismos, se construyeron 

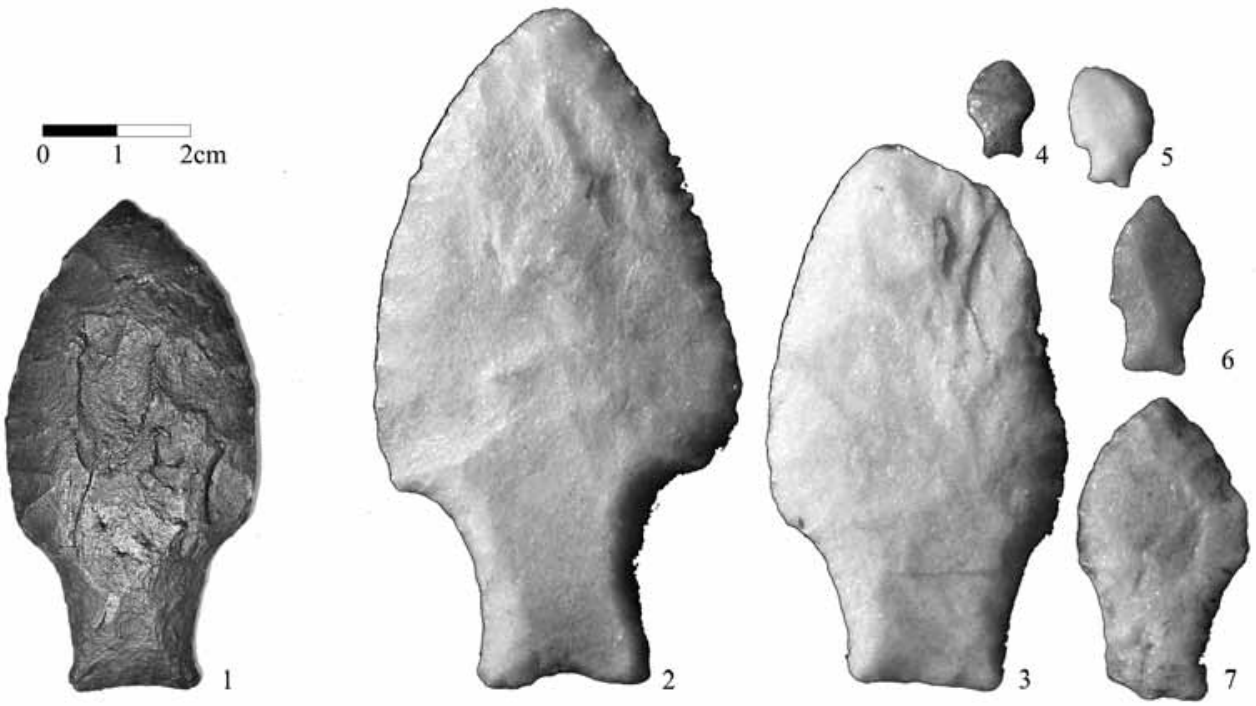

Fig. 1. Variabilidad morfológica de artefactos con diseño de puntas cola de pescado 1 Cueva Fell, 2 y 3, puntas grandes de Cerro El Sombrero Cima. 4, 5 y 6 puntas pequeñas de Cerro El Sombrero Cima y 7 punta mediana de Cerro El Sombrero Cima.
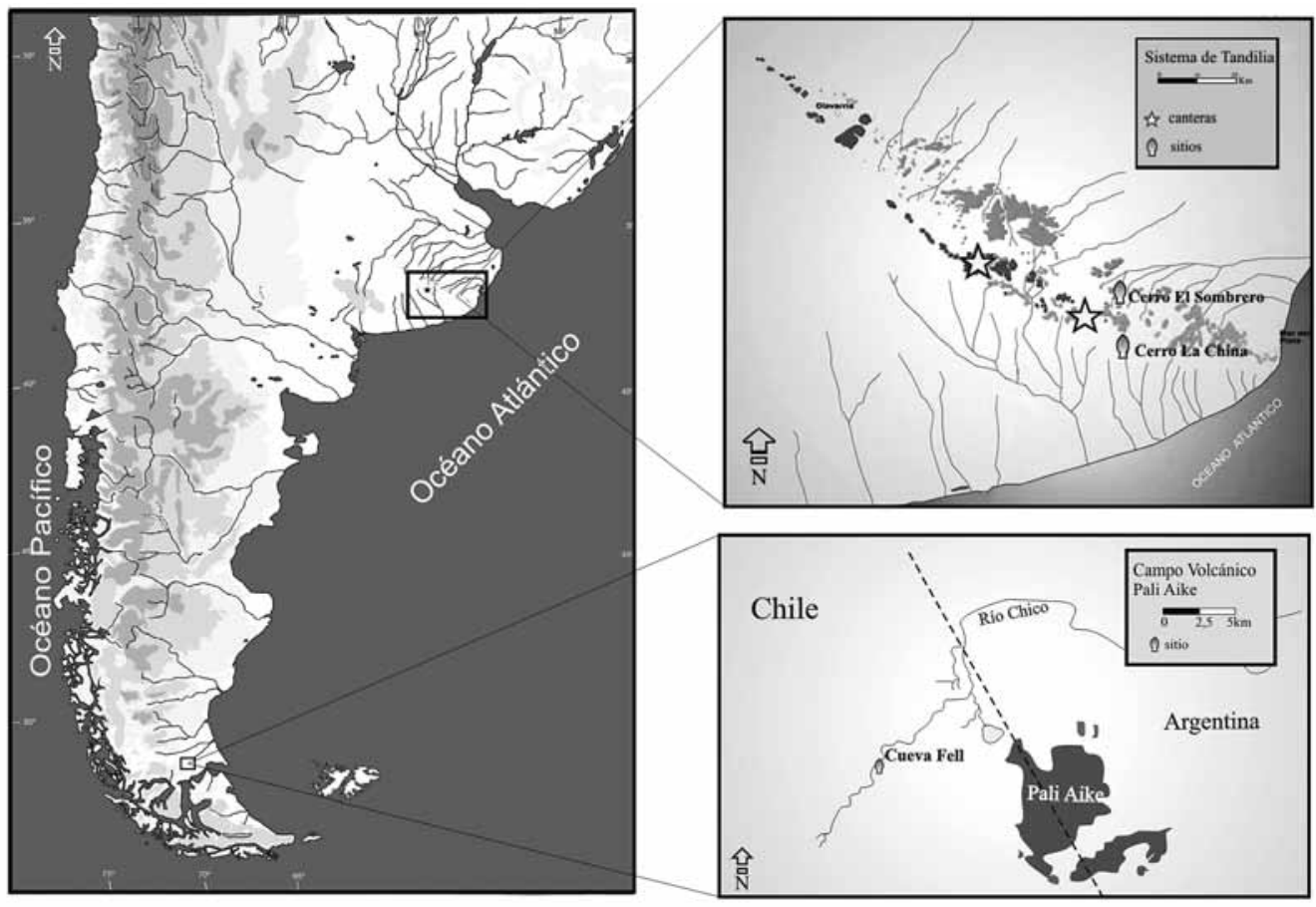

Fig. 2. Mapa de localización de los sitios arqueológicos estudiados. 1, Localidad Cerro El Sombrero; 2, Localidad Cerro La China; 3, Sitio Cueva Fell. 
varios modelos interpretativos para las diversas combinaciones posibles de atributos de los desechos. En dichas construcciones se priorizaron los siguientes aspectos:

1. La identificación de un conjunto mínimo de atributos a ser registrado en cada desecho, lo suficientemente objetivo como para no ser malinterpretado por y entre los analistas (Sullivan y Rozen 1985; y discusión alli citada).

2. La combinación de una aproximación formal (como la mencionada en el punto 1) con un enfoque que permita analizar los conjuntos de desechos producidos a partir del trabajo de un mismo bloque de roca como una unidad (Frison 1974; Kelly 1985; Larson 1994; Larson \& Kornfeld 1997). Este desarrollo experimental, generado en el ámbito de la University of Wyoming es utilizado en este trabajo.

3. El empleo de modelos que utilizan funciones matemáticas que permiten el estudio de conjuntos de desechos como parte de un continuo (Ahler 1989; Shott 1994; Larson 1994; Ingbar et al. 1989).

Es decir, el enfoque de análisis que aquí aplicamos reconoce sus antecedentes en una línea de trabajo que comienza con la propuesta de G. Frison de definir grupos de desechos sobre las bases del color, textura y tipo de roca previo a cualquier estudio (Frison 1974). En 1985, al aplicar este método, Kelly agrega el registro de tipo de grano, inclusiones, pátina y evidencias de tratamiento térmico. Siguiendo esta línea de investigación, Larson y Kornfeld proponen en 1997 el Minimum Analytical Nodule Analysis (MANA) o análisis de nódulos mínimos, como forma de separar los artefactos procedentes de un mismo nódulo, ya sean estos lascas, desechos, núcleos o instrumentos y realizan inferencias a partir de cómo están constituidos esos conjuntos.

En cuanto al primer paso metodológico, pensamos que, reconocer y aislar el número mínimo de grupos de desechos pertenecientes a un mismo conjunto lítico y por ende inferir eventos finitos de talla, es de gran utilidad como paso previo a cualquier estudio de técnicas de manufactura y/o asignación funcional de los asentamientos. Asimismo, la aplicación de esta metodología resulta adecuada para las divisiones de los conjuntos de materiales líticos de algunos contextos de Pampa y Patagonia debido al uso prehistórico de nódulos y/o afloramientos con rocas con características distintivas, cuyos minerales les otorgan coloraciones y texturas particulares. En el caso pampeano, las ortocuarcitas - materia prima dominante en los conjuntos artefactuales- exhiben características macroscópicas semejantes en las fuentes de abastecimiento y en la mayoría de los sitios de la región, lo cual dificulta la identificación de núcleos distintos. Sin embargo, en los sitios aquí estudiados la selección y uso de variedades con diferentes colores por parte de los pobladores (Flegenheimer \& Bayón 1999), ha facilitado la identificación de nódulos mínimos. Asimismo, las rocas volcánicas negras de Cueva Fell en Patagonia o la variedad de ortocuarcuarcita blanca del Grupo Sierras Bayas, en la región pampeana encuentran un grado mayor de dificultad para su separación por nódulos. Si bien este hecho redujo las posibilidades de división en algunas rocas en el caso de estudio, y por lo tanto disminuyó el número mínimo de eventos identificado, se han podido reconocer ciertas diferencias en observaciones con lupa que permitieron separar diferentes eventos. Según la propuesta de Larson \& Kornfeld (1997), separar dichos eventos de talla estudiando detenidamente la materia prima es sólo el primer paso, luego se procede a ubicar los instrumentos y núcleos dentro de estos conjuntos. Este trabajo con los conjuntos permite realizar interpretaciones de una indole distinta y de mayor detalle que las aquí tratadas. En Argentina este enfoque ha sido aplicado de forma completa a cuarzos y sílices del centro del país y se compararon los resultados obtenidos en estas nuevas experimentaciones con los ya conocidos para otras materias primas y técnicas de talla (Sario 2007, 2009; Sario \& Pautassi 2012; Pautassi \& Sario 2011). Estos trabajos permitieron discutir las variaciones en el modelo en relación al tipo de materia prima, la técnica de talla y la habilidad del tallador.

En cuanto al análisis no tipológico, el segundo paso metodológico aquí aplicado, para abordar el punto 1 (ver supra) se utilizaron atributos de carácter ordina vinculados a mediciones (largo, ancho, espesor, cantidad de lascados previos sobre la cara dorsal). Es decir, se eligió un método de análisis que minimice la variabilidad de las observaciones intra e inter observador (para más datos sobre estas discusiones ver Dibble \& Bernard 1980; Sullivan \& Rozen 1985; Shott 1994; Dibble 1997). A partir de la base de estas discusiones acerca del error o variabilidad de las observaciones, se priorizaron 
aquellos atributos que pudieran medirse una y otra vez y que incluso permiten cuantificar el grado de error. Así, sobre la base de trabajos experimentales, Ingbar y colaboradores (1989) y Shott (1996) concluyeron que los atributos donde se registra menor error son: peso de la lasca, cantidad de negativos de lascados anteriores registrados por las aristas en la cara dorsal, largo total de la lasca, ancho total de la lasca, espesor en la sección transversal, ancho de la plataforma de percusión, largo de la plataforma de percusión y cantidad de negativos de lascados anteriores en la plataforma de percusión.

A partir de la combinación de estos nueve atributos estos autores desarrollaron cinco modelos con una base experimental para evaluar el momento (más temprano o más tardío) en el proceso de reducción en el que se extrajo una lasca en particular; en el planteo original se trabajó tanto con núcleos como con bifaces y utilizando distintas técnicas de producción. Así, la talla es considerada en estos modelos como un proceso continuo, y cada desecho se analiza para determinar el momento en el que se predice que fue tallado.

Siguiendo a Ingbar et al. (1989), cada uno de los modelos considera una curva de regresión. Dichas curvas sirven entonces para predecir, con diferentes grados de adecuación, cuándo se extrajo un desecho en particular. En este trabajo elegimos utilizar la fórmula del Modelo 4 (Ingbar et al 1989:126) para realizar el análisis; este presenta una $\mathrm{R}^{2}$ de 0,94 . La misma se establece sobre la base de las siguientes relaciones:

$$
\begin{array}{r}
y_{\text {predicho }}=-12.14 x(\log E)+9.65 x(\log \text { DLD }) \\
\text { Donde: } \log =\text { Logaritmo; } E=\text { espesor }
\end{array}
$$

de la lasca; DLD = densidad de lascados dorsales (cantidad de negativos de lascados en el dorso/ ancho x largo de la pieza).

Es importante destacar que los conjuntos de desechos de talla arqueológicos son considerados como el palimpsesto de eventos y técnicas de manufactura y que, como tal fueron entendidos por Ingbar y colaboradores a la hora de desarrollar su modelo. En nuestro análisis de desechos de talla aplicamos este modelo para cada una de las materias primas presentes, es decir, luego de separar los desechos por nódulo mínimo. Esto nos permite evaluar con posterioridad si existen diferencias significativas en las actividades de talla entre los sitios y también de cada uno de los nódulos de distintas materias primas dentro del mismo sitio. Sin embargo, cabe destacar que la unidad de análisis en estos estudios es el desecho de talla y los conjuntos son construcciones interpretativas.
Pretendemos entonces, aplicar una metodología de estudio a conjuntos de desechos líticos que permita estimar el momento en el cuál fue extraído cada desecho. Esto nos permite aproximarnos a un aspecto de las actividades de talla practicadas en los sitios para comparar y discutir los procesos de manufactura en distintos espacios con ocupaciones coetáneas.

\section{LAS LOCALIDADES ARQUEOLÓGICAS}

En las localidades arqueológicas de las dos regiones que consideramos, se han estudiado ocupaciones asignadas a grupos humanos que usaron puntas de proyectil cola de pescado o Fell 1 durante la transición Pleistoceno - Holoceno. En ambas regiones se identificaron varios sitios vinculados entre sí. En tal sentido, las ocupaciones tempranas de la Patagonia meridional chilena han sido recientemente analizadas por Jackson (2007) quien llevó a cabo un estudio comparativo entre las estructuras arqueológicas, la intensidad y la reiteración de las ocupaciones, este estudio consideró las evidencias líticas. Así, el sitio Cueva Fell se integraría con Cueva Palli Aike en un área de circulación (Jackson 2007). Para el caso de la región Pampeana la zona ha sido considerada como una microregión (sensu Aschero 1988) y se han correlacionado las ocupaciones de los distintos sitios y las áreas de abastecimiento de rocas. Por ejemplo, Cerro La China y Cerro El Sombrero se han integrado en un sistema de asentamiento con gran variabilidad intersitio en el que se ocuparon diferentes espacios con distintas funcionalidades (Flegenheimer 2004). También recientemente se ha analizado la zona desde la perspectiva de la arqueología del paisaje y se consideraron las posibles redes de comunicación entre estos espacios (Mazzia 2010-2011; Mazzia \& Flegenheimer 2012).

\section{LOCALIDAD CERRO EL SOMBRERO}

La localidad Cerro El Sombrero está ubicada en la región pampeana, en el sector centro-este del Sistema de Tandilia. Se trata de un cerro mesa de $429 \mathrm{~m}$, el más alto de la zona, que se destaca unos $200 \mathrm{~m}$ por sobre la llanura circundante (Fig 3.1). En este cerro se realizaron excavaciones a cielo abierto en la cima, donde se encuentra el sitio más denso de los aquí analizados, y en un abrigo que se abre en las rocas que rodean la cima (Flegenheimer 2003, 2004). 


\section{Sitio Cerro El Sombrero Cima}

Cerro El Sombrero Cima presenta una superficie de aproximadamente $25.000 \mathrm{~m}^{2}$, la mitad central de la cual está cubierta por sedimentos de loess cuaternarios rodeados por afloramientos de ortocuarcitas que forman un pequeño acantilado alrededor de la cima. El material arqueológico se encuentra tanto sobre la superficie, como incluido en los sedimentos que exhiben espesores variados y conforman diferentes secuencias estratigráficas en los distintos sectores del sitio (Fig. 3.2). Las excavaciones hasta el momento ocupan menos del $1 \%$ de la superficie disponible y en la mayoría de los sectores excavados se ha recuperado material lítico que presenta gran homogeneidad en cuanto a sus características técnicas, tipológicas y estado de integridad. Aunque la mala preservación de la materia orgánica no favoreció la conservación de muestras para fechar, dada la homogeneidad y las características del conjunto lítico, la ocupación se adjudicó a la transición Pleistoceno - Holoceno por comparación con otros sitios cercanos fechados por radiocarbono (Zárate et al. 2000-2002; Flegenheimer 2003, 2004; Flegenheimer \& Mazzia 2013).

Las excavaciones abarcan $37 \mathrm{~m}^{2}$ y se encuentran distribuidas a lo largo y ancho de la cima del cerro. En las mismas, salvo una cuadrícula donde sólo se recuperaron 4 lascas, se registró una densidad de artefactos que va desde dos artefactos formatizados y cuatro lascas con talón por $\mathrm{m}^{2}$, hasta 46 artefactos formatizados y 145 lascas con talón por $\mathrm{m}^{2}$, en las cuadrículas más densas. Resulta llamativo el alto porcentaje de fractura de los artefactos formatizados (90\%) y la elevada frecuencia de bifacialidad (Flegenheimer 1991, 1999, 2003; Weitzel \& Flegenheimer 2007; Weitzel 2010). La colección de excavación reúne 30 puntas de proyectil cola de pescado, en su mayoría fragmentadas, siete de ellas son preformas y cinco se encuentran recicladas. Están asociadas a una variedad de instrumentos tallados $(\mathrm{n}=617)$ que incluyen raederas, artefactos mediano pequeños de bisel oblicuo, raclettes, artefactos con retoque sumario, raspadores, cuchillos, bifaces y esbozos de piezas bifacales, artefactos con puntas destacadas y cortantes, aunque en su mayoría no se pueden identificar por estar fracturados. También incluye artefactos manufacturados por picado, abrasión y pulido fragmentados: piedras discoidales y esferas.
En cuanto a los desechos, estos no conservaron corteza y su rango de tamaño oscila entre muy pequeño y mediano pequeño (según Aschero 1975); una gran cantidad son producto del adelgazamiento bifacial (19\%) y se los interpretó como resultado de los eventos finales de las cadenas de manufactura (Flegenheimer 1988, 1991). En este trabajo se empleó una muestra de desechos recuperada en 13 $\mathrm{m}^{2}$ del sector más denso del sitio.

La materia prima predominante en los instrumentos de Cima (94\%) es la ortocuarcita Grupo Sierras Bayas (GSB), cuyas canteras se encuentran a una distancia de 40-60 km del sitio (Bayón et al. 2006; Colombo \& Flegenheimer 2011). También se ha empleado un escaso $3 \%$ de cuarzos, que afloran junto con la ortocuarcita GSB y en las inmediaciones del sitio, y el 3\% restante incluye rocas indeterminadas, calizas silicificadas, diabasa, ftanita, dacita y dolomía silicificada (Bayón et al. 2006).

En base a la evidencia de manufactura de puntas y la gran cantidad de pedúnculos recuperados en este sitio, se han propuesto actividades de reparación y re-equipamiento del sistema de armas; en cuanto a la talla, estas tareas requirieron de acciones vinculadas a los últimos momentos de manufactura (Flegenheimer 1988, 2003, 2004). Asimismo, algunos de los artefactos descartados en la cima son poco frecuentes o no están presentes en los sitios vecinos y recientemente se ha propuesto que en este lugar posiblemente se descartaron artefactos fracturados en otros sitios (Weitzel 2010). Por otro lado, la vista panorámica desde la cima del cerro lo convierte en un excelente avistadero; esta situación debió ser de especial importancia para tener un buen control del territorio y es uno de los rasgos considerados relevantes para interpretarlo como un lugar particular con un papel significativo en el pasado (Flegenheimer 1988, 2003; Mazzia \& Flegenheimer 2012; Flegenheimer \& Mazzia 2013).

\section{Sitio Cerro El Sombrero Abrigo 1}

Este abrigo, excavado en la ortocuarcita que rodea la cumbre, se encuentra hacia el norte del sitio Cima, a unos 10 minutos de caminata. Las excavaciones en el mismo ocupan $12 \mathrm{~m}^{2}$ pero la superficie disponible durante el momento de la ocupación era el doble (Fig. 3.3 y 3.4). Se han distinguido dos unidades estratigráficas y los 
materiales arqueológicos están concentrados en la Unidad 1 o inferior. Cuatro pequeñas muestras de carbón recuperadas entre los restos de ocupación han brindado edades entre $10.725 \pm 90$ y 10.270 $\pm 85 \mathrm{AP}$ y un quinto fechado más reciente es considerado anómalo (Flegenheimer \& Zárate 1997; Flegenheimer 2003). La excavación brindó dos puntas de proyectil cola de pescado, una entera y otra fracturada y 25 instrumentos, entre ellos: raederas, artefactos medianos pequeños de bisel oblicuo, raclettes, cuchillos y bifaces. También se hallaron seis núcleos, desechos de talla y fragmentos de ocre (Flegenheimer 2003, 2004).

La mayoría de los instrumentos del sitio están confeccionados sobre ortocuarcita GSB (79\%), sin embargo, también hay un porcentaje importante de rocas inmediatamente disponibles talladas, principalmente cuarzo (16\%), porcentaje que es mayor entre los desechos. Los instrumentos se tallaron por retoque unifacial, bifacial y talla bipolar. En este sitio se han identificado dos trayectorias principales en el tratamiento de las rocas: aquéllas recolectadas en las inmediaciones presentan una menor inversión de trabajo, con mayor presencia de las primeros momentos de manufactura llevados a cabo en el sitio, y las que provienen de canteras más alejadas en cambio, que se emplearon en la talla de los artefactos más elaborados y entraron a éste y a otros sitios, bajo la forma de núcleos y/o de artefactos ya formatizados (Bayón et al. 2006).

Dada la variedad de artefactos recuperados y la existencia de piezas correspondientes a distintas etapas de manufactura, inicialmente se consideró que en este sitio la gente desarrolló varias actividades, posiblemente domésticas. Estudios microscópicos posteriores han permitido precisar esta interpretación y ahora se lo concibe como un sitio de actividades específicas, donde la tarea predominante se
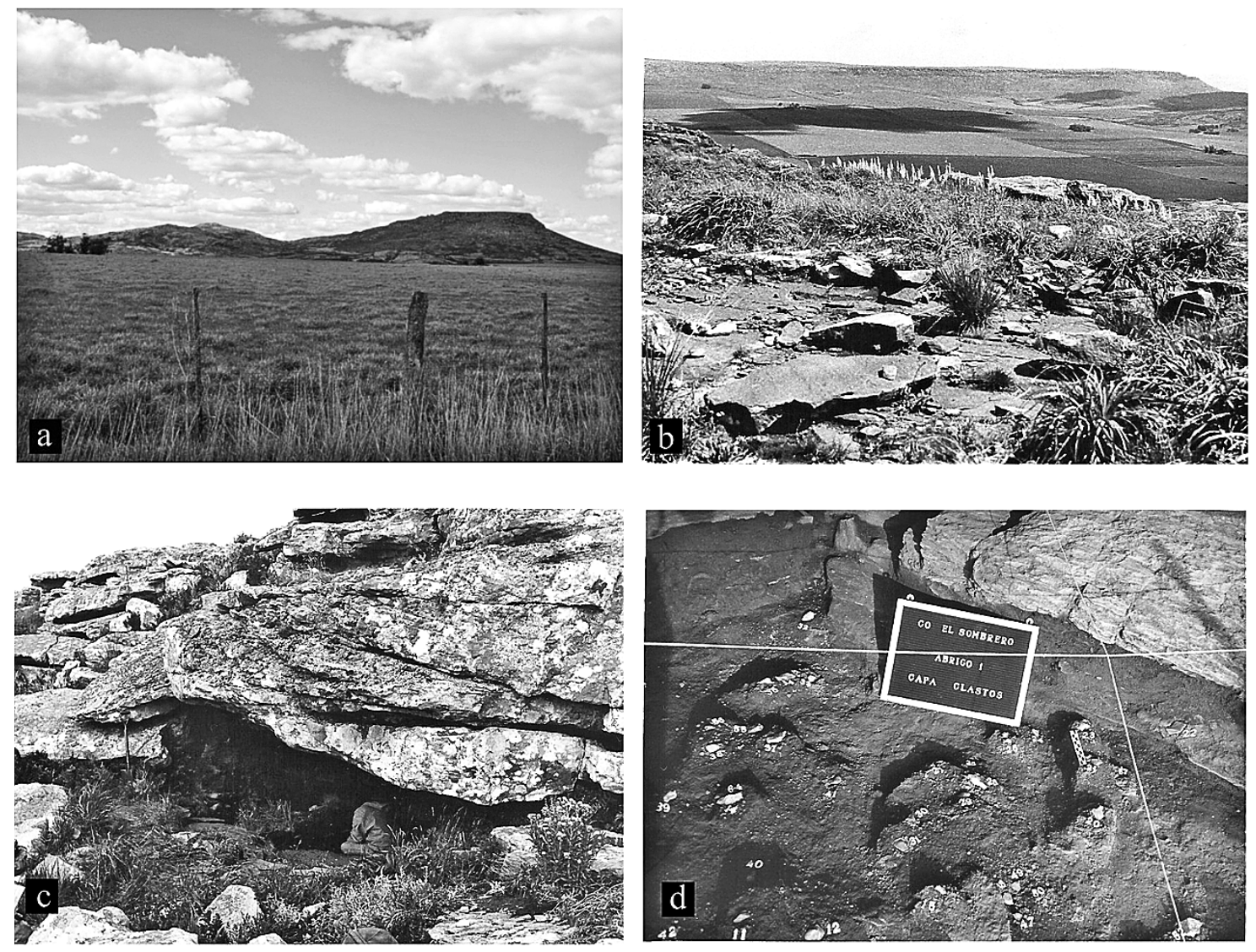

Fig. 3. a) Vista panorámica de la Localidad Cerro El Sombrero.

b) Excavaciones en Localidad Cerro El Sombrero-sitio Cima

c) Vista de Cerro El Sombrero-sitio Abrigo 1. d) Detalle de las excavaciones sitio Abrigo 1. 
correspondería con las primeras etapas del trabajo de pieles en estado fresco (Flegenheimer 2003; Flegenheimer \& Leipus 2007).

\section{LOCALIDAD CERRO LA CHINA, SITIO 1}

Este sitio se encuentra en un pequeño abrigo localizado en un cerro bajo (Fig. 4.1). Se excavó en el abrigo, en el talud y en sus inmediaciones, en un área total de $18 \mathrm{~m}^{2}$ (Flegenheimer 2004). Se distinguieron dos unidades estratigráficas fértiles y dos inferiores, estériles. La unidad superior comprende ocupaciones del Holoceno medio y tardío, que resultan indistinguibles entre sí, y en la unidad 3 se recuperaron los materiales aquí estudiados, fechados entre $10.525 \pm 75$ y 10.804 \pm 75 AP (Flegenheimer 2004; Flegenheimer \& Zárate 1997; Mazzia \& Flegenheimer 2007).

En las ocupaciones tempranas de este sitio, así como en el Abrigo 1 ya mencionado, se identificaron varias trayectorias en el uso de las materias primas. Las rocas inmediatamente disponibles, que en este caso son las ortocuarcitas de la Formación Balcarce (FB), fueron empleadas en la confección de instrumentos poco elaborados cuya manufactura se debió realizar en el sitio, ya que se recuperaron núcleos y lascas grandes, con reserva de corteza. En cambio, la mayor parte del instrumental fue tallado sobre ortocuarcita GSB que debió entrar al sitio principalmente como formas base o instrumentos tallados con anterioridad, un escaso número de núcleos abre la posibilidad de actividades de extracción mínimas. Asimismo, un pequeño porcentaje de instrumentos elaborados en calizas silicificadas fueron trasladados desde largas distancias (Bayón et al. 2006).

Los instrumentos incluyen raederas, artefactos mediano pequeños de bisel oblicuo, cuchillos, puntas destacadas, etc. y fueron confeccionados por retoque unifacial, bifacial y talla bipolar (Flegenheimer 2004). Entre los artefactos bifaciales se identificó una preforma de cola de pescado fracturada. Tanto la variedad de artefactos y de actividades de talla, como las condiciones de reparo y accesibilidad del sitio han conducido a pensar que se trataba de un entorno doméstico donde se desarrollaron distintas tareas, incluso la manufactura de puntas.

\section{SITIO CUEVA FELL}

Este abrigo rocoso de cerca de $61 \mathrm{~m}^{2} \mathrm{se}$ encuentra ubicado en el valle del río Chico, en territorio de la Patagonia chilena (Fig. 2 y 5.1 a 5.3). El área excavada, de acuerdo a Jackson (2007), es de $15 \mathrm{~m}^{2}$. Es una de las localidades arqueológicas estudiadas por J. Bird a partir de 1936 (Bird 1938, 1988) y desde el comienzo de las investigaciones ha sido considerada como una de las evidencias de ocupación temprana del continente americano, caracterizándose como Modalidad Cultural Fell 1 (sensu Massone \& Prieto 2004).

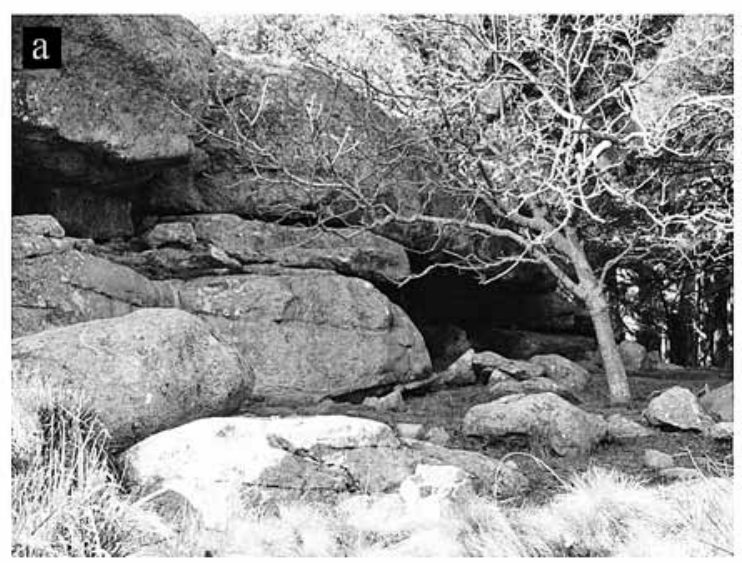

Fig. 4. Vista general de sitio Cerro La China- sitio1.

J. Bird interpretó la existencia de cinco niveles arqueológicos (I a V), y en el más antiguo de ellos, nivel I, registró quince puntas de proyectil, algunas de ellas acanaladas, a las que denominó "colas de pescado" por su morfología (luego denominadas también puntas tipo Fell por su lugar de hallazgo). Asociadas a ellas aparecen otros instrumentos, lascas y desechos de talla así como también innumerables restos de fauna que incluyen mamíferos extinguidos. Entre los instrumentos asociados hay un conjunto de cuarenta y cinco artefactos de piedra tallados (Bird 1938, 1988) entre los que se identificaron: raspadores, raederas y cuchillos. También hay un conjunto de instrumentos manufacturados por picado abrasión y pulido: los litos discoidales (Bird 1938; Jackson \& Méndez 2007). Esta primera colección se encuentra hoy en el American Museum of Natural History. En 1959 Reichlen \& A. Lamming-Emperaire (Emperaire et al. 1963; Laming Emperaire 1967) encuentran cuatro puntas más. Entre 1953 y 1960, John Fell realizó diversas observaciones, limpiezas de perfiles y excavaciones, a instancias de Bird quien deseaba obtener una muestra de carbón en los depósitos 
de la primera ocupación para poder determinar su antigüedad. Así, en 1960 Fell obtuvo una muestra de carbón que dio una edad de $10.720 \pm 300 \mathrm{AP}$, para el período I del sitio. Estas recolecciones y excavaciones posteriores de John Fell conformaron una nueva colección alojada en el Centro de Estudios del Hombre Austral del Instituto de la Patagonia. Los estudios recientes e interpretaciones sobre los materiales de esta localidad y los alrededores pueden consultarse en varias publicaciones (Massone 1981, 1996; Nami 1993-94, 2003; Schidlowsky 2001; Massone \& Prieto 2004; Charlin 2005, 2006, 2007 a y b, 2009; Jackson \& Prieto 2005; Jackson 2007). Para una historia de las trayectorias de investigación sobre este sitio ver Massone (1981, 1996) y Massone \& Prieto (2004).

Luego del estudio de ambas colecciones líticas, del American Museum of Natural History y del Instituto de la Patagonia, Nami reconoce como evidencias de la manufactura de las puntas de proyectil cola de pescado una probable preforma y cuatro formas base bifaciales en etapas tempranas de manufactura (Nami 1997, 1999, 2003, 2010). En base a estos trabajos este autor sugiere que en este sitio se llevaron a cabo actividades de reparación de armas y reemplazo de puntas. En cuanto a las materias primas líticas, las predominantes fueron designadas originalmente como basaltos (Bird 1938, 1988). Las mismas se encuentran disponibles en un rango de distancia local menor a $40 \mathrm{~km}$ (Jackson 2007) y recientemente las variedades de rocas de grano fino oscuras fueron agrupadas genéricamente como RGFO por Charlin
(2005, 2007a). Las mismas incluyen a los basaltos, vitrófiros dacíticos, andesita, ftanita, lutita, grauvaca y limonita. Esta autora menciona, sin embargo, que el basalto ha sido la roca predominantemente utilizada para la confección de las puntas de proyectil.

\section{RESULTADOS}

Los resultados presentados a continuación provienen del estudio de lascas enteras de los sitios mencionados. En el caso de Cerro El Sombrero Cima sólo se ha analizado una muestra dada la gran cantidad y homogeneidad de los desechos; en cambio, en Cerro El Sombrero Abrigo 1 y Cerro La China Sitio 1 se analizaron el total de las lascas enteras recuperadas. Los desechos de talla de las colecciones de Cueva Fell no habían sido previamente analizados, de modo que este estudio constituye una primera aproximación. Las cifras totales pueden observarse en la Tabla 1 donde también se señala el número de eventos reconocidos, es decir, el número de nódulos mínimos integrado por lascas de una misma materia prima, y también, la cantidad de lascas que integra cada uno de estos nódulos.

Para el caso de los sitios de la región pampeana los eventos con mayor número de desechos corresponden a la ortocuarcita GSB blanca. Esto se debe a que como dijimos, las rocas blancas no poseen caracteres distintivos que permitan desagregar con gran detalle los distintos eventos de talla. En cambio, en las ortocuarcitas coloreadas pudieron identificarse mayor número de nódulos. También para
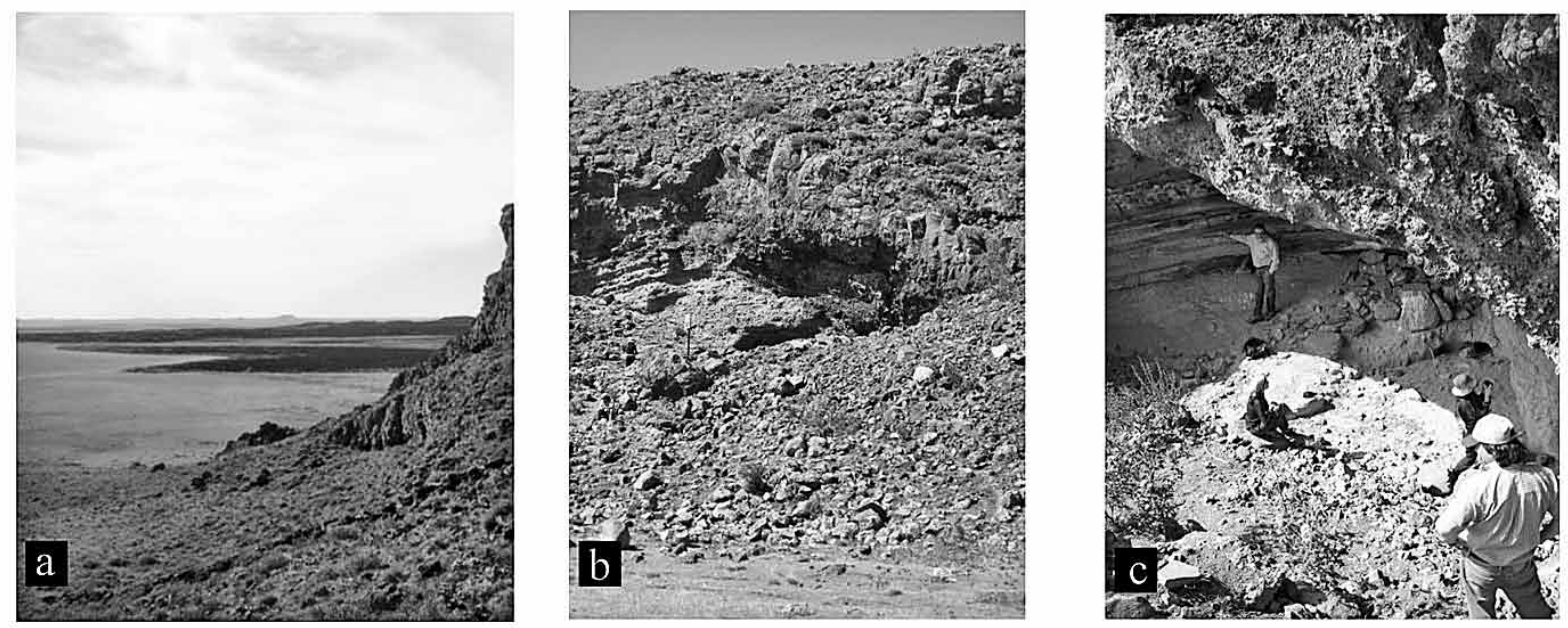

Fig. 5. a) Vista general del campo volcánico de Pali Aike y b) y c) sitio Cueva Fell (Fotografías Dr. Ramiro Barberena). 
Tabla 1. Datos de la muestra estudiada por sitio arqueológico, tipo de materia prima y cantidad de conjuntos interpretados siguiendo la propuesta de Frison (1974), Aschero (1975) y Larson \& Kornfeld (1997).

\begin{tabular}{|c|c|c|c|c|}
\hline Sitio & Materia prima & $\begin{array}{c}\text { Cantidad } \\
\text { de desechos } \\
\text { analizados }\end{array}$ & $\begin{array}{l}\text { Cantidad } \\
\text { de eventos } \\
\text { reconocidos }\end{array}$ & Lascas por nódulo \\
\hline \multirow{6}{*}{$\begin{array}{l}\text { Cerro El Sombrero } \\
\text { Cima }\end{array}$} & Ortocuarcita GSB & 179 & 33 & $\begin{array}{c}51,28,10,9,9,8,7,6,6,5,4,3,3,3,2,2,2,2,2, \\
2,2,2,1,1,1,1,1,1,1,1,1,1\end{array}$ \\
\hline & Ortocuarcita FB & 2 & 2 & 1,1 \\
\hline & Cuarzo & 1 & 1 & 1 \\
\hline & $\begin{array}{c}\text { Caliza silicificada } \\
\text { colorada }\end{array}$ & 1 & 1 & 1 \\
\hline & Ftanita & 2 & 2 & 1,1 \\
\hline & Total & 185 & 39 & \\
\hline \multirow{6}{*}{$\begin{array}{l}\text { Cerro El Sombrero } \\
\text { Abrigo } 1\end{array}$} & Ortocuarcita GSB & 85 & 21 & $\begin{array}{c}20,18,7,6,5,4,4,3,3,2,2,2,2,1,1 \\
1,1,1,1,1,1\end{array}$ \\
\hline & Ortocuarcita FB & 10 & 6 & $3,2,2,1,1,1$ \\
\hline & Cuarzo & 60 & 7 & $34,16,4,4,3,1,1$ \\
\hline & Ftanita & 3 & 1 & 3 \\
\hline & Otros & 9 & 5 & $4,2,1,1,1$ \\
\hline & Total & 167 & 40 & \\
\hline \multirow{6}{*}{$\begin{array}{c}\text { Cerro La China } \\
\text { Sitio } 1\end{array}$} & Ortocuarcita GSB & 113 & 13 & $44,18,16,14,10,2,2,2,2,1,11,1$ \\
\hline & $\begin{array}{l}\text { Ortocuarcita FB } \\
\text { Cuarcita Balcarce }\end{array}$ & 26 & 3 & $21,4,1$ \\
\hline & Caliza silicificada & 2 & 2 & 1,1 \\
\hline & Diabasa & 1 & 1 & 1 \\
\hline & Otros & 1 & 1 & 1 \\
\hline & Total & 143 & 20 & \\
\hline \multirow{12}{*}{$\begin{array}{l}\text { Cueva Fell } \\
\text { I. de la P }\end{array}$} & RGFO & 103 & 1 & 103 \\
\hline & Cuarzo & 4 & 1 & 4 \\
\hline & Vulcanita Verde & 16 & 1 & 16 \\
\hline & Calcedonia & 18 & 1 & 18 \\
\hline & $\begin{array}{l}\text { Silícea no ident. } 1 \\
\text { identificada }\end{array}$ & 3 & 1 & 3 \\
\hline & Ópalo & 4 & 1 & 4 \\
\hline & Silícea no ident. 2 & 6 & 1 & 6 \\
\hline & Cuarcita & 6 & 1 & 6 \\
\hline & Cuarzo Gris & 8 & 1 & 8 \\
\hline & Cuarzo otro color & 1 & 1 & 1 \\
\hline & Silícea no ident. 3 & 1 & 1 & 1 \\
\hline & Cuarcita Gris & 1 & 1 & 1 \\
\hline \multirow{3}{*}{ Cueva Fell } & RGFO & 208 & 1 & 208 \\
\hline & Cuarzo & 9 & 1 & 9 \\
\hline & Vulcanita Verde & 9 & 1 & 9 \\
\hline Total & & 397 & 13 & \\
\hline
\end{tabular}


el caso de Cueva Fell ha sido muy difícil identificar eventos discretos dentro de cada materia prima, particularmente en el caso del basalto o RGFO, no pudieron identificarse diferencias macroscópicas que permitan separar nódulos.

A continuación se discuten tanto los resultados del MANA como los resultados obtenidos por sitio al aplicar el Modelo 4 propuesto por Ingbar y colaboradores (Ingbar et al. 1989). En los gráficos siguientes (Figs. 6 a 10) se encuentran representados los distintos nódulos discriminados por materia prima identificados en cada sitio, en los mismos se puede observar qué trayecto de la secuencia de manufactura representa cada lasca en cada caso.

\section{SITIO CERRO EL SOMBRERO CIMA}

En Cerro El Sombrero Cima se pudo identificar un total de 39 eventos distintos de talla para una muestra de 185 desechos (Tabla 1 y Fig. 6). Como puede observarse, en todos los casos las curvas comienzan después de la extracción 60, esto indica que los desechos de talla corresponden a momentos avanzados de la manufactura, cuando ya se obtuvieron aproximadamente más de 60 lascas previas. En todos los nódulos están ausentes los desechos correspondientes a los primeros momentos de la manufactura, salvo un caso que corresponde a una lasca primaria. Asimismo, en el conjunto sólo se registran extracciones de hasta 110 lascas por nódulo aproximadamente. La ausencia de extracciones posteriores puede deberse a varias razones, entre ellas: que éstas se llevaran a cabo en otro sitio, que un gran número de desechos de tamaños muy pequeños se encuentran fracturados y no pudieron ser estudiados con esta metodología, o más probablemente a que la manufactura/reciclaje de los artefactos tallados en la cima (bifaces, puntas) no requirió de más extracciones.

Aquellos eventos que incluyen el mayor número de desechos (todos de ortocuarcita GSB) presentan promedios que van desde la extracción número 59 a la 81 (Tabla 2). Este dato es relevante para la comparación entre los conjuntos líticos de los distintos sitios. Los eventos de otras materias primas en Cima son escasos y siguen la tendencia a ser posteriores a la extracción número 60 .
Tabla 2. Promedio de momento de extracción para los eventos de talla de la ortocuarcita Grupo Sierras Bayas del sitio Cerro El Sombrero Cima.

\begin{tabular}{|c|c|c|}
\hline $\begin{array}{c}\text { Nombre } \\
\text { del evento }\end{array}$ & Promedio & $\begin{array}{c}\text { Desviación } \\
\text { estándar }\end{array}$ \\
\hline OGSB E1 & 75,51 & 10,84 \\
\hline OGSB E3 & 81,71 & 13,40 \\
\hline OGSB E5 & 72,98 & 12,30 \\
\hline OGSB E 7 & 70,70 & 7,96 \\
\hline OGSB E12 & 68,34 & 9,63 \\
\hline OGSB E20 & 75,05 & 11,50 \\
\hline OGSB E22 & 62,98 & 4,12 \\
\hline OGSB E23 & 73,59 & 12,87 \\
\hline OGSB E24 & 74,03 & 6,77 \\
\hline OGSB E26 & 58,94 & 9,92 \\
\hline
\end{tabular}

\section{SITIO CERRO EL SOMBRERO ABRIGO 1}

Se estudió una cantidad similar de desechos a los del sitio Cima y se identificó una cantidad similar de eventos: 167 desechos y se recuperaron 40 eventos de talla, de los cuales 21 corresponden a ortocuarcita GSB (Tabla 1). Un vez que se aplica el modelo de Ingbar y colaboradores, se observan dos curvas de regresión distintas, las que corresponden por un lado, a las rocas que están inmediatamente disponibles y por otro, a todas las restantes (Fig.7).

La mayor frecuencia de los eventos de talla comienza alrededor de la extracción número 50 , sin embargo para el cuarzo y la ortocuacita FB (inmediatamente disponibles) también se registran lascas que corresponden a la primera extracción. Como puede observarse en la tabla 3 esto afecta la desviación estándar del promedio, arrojando valores elevados. La mayoría de los eventos finalizan hacia la extracción número 120.

La ortocuacita GSB presenta un número promedio de extracciones entre 66 y 70 (Tabla 3). En cambio, el cuarzo da promedios más bajos de entre 54 y 63 extracciones, pero estos promedios se encuentran altamente influidos por las lascas de los primeros momentos tal como puede verse en la tabla 3 de las desviaciones estándar. En la figura 7 resulta notoria la ausencia de desechos entre el momento inicial y las extracciones alrededor del número 50 , tema que discutiremos más adelante. 


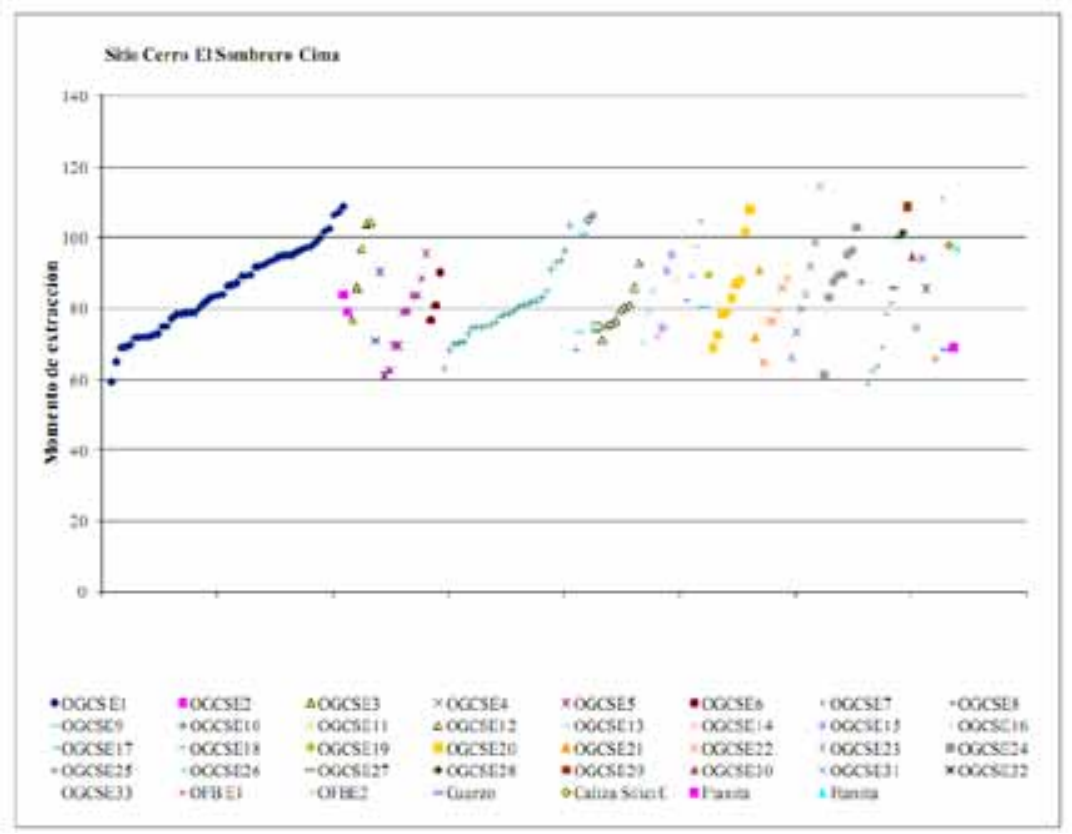

Fig. 6. Resultado del análisis no tipológico obtenido al aplicar el Modelo 4 (Ingbar et al. 1989) para las lascas del sitio Cerro El Sombrero Cima. Cada conjunto con la misma referencia representa los desechos de talla correspondientes a un mismo nódulo mínimo y cada punto corresponde a un desecho de talla.

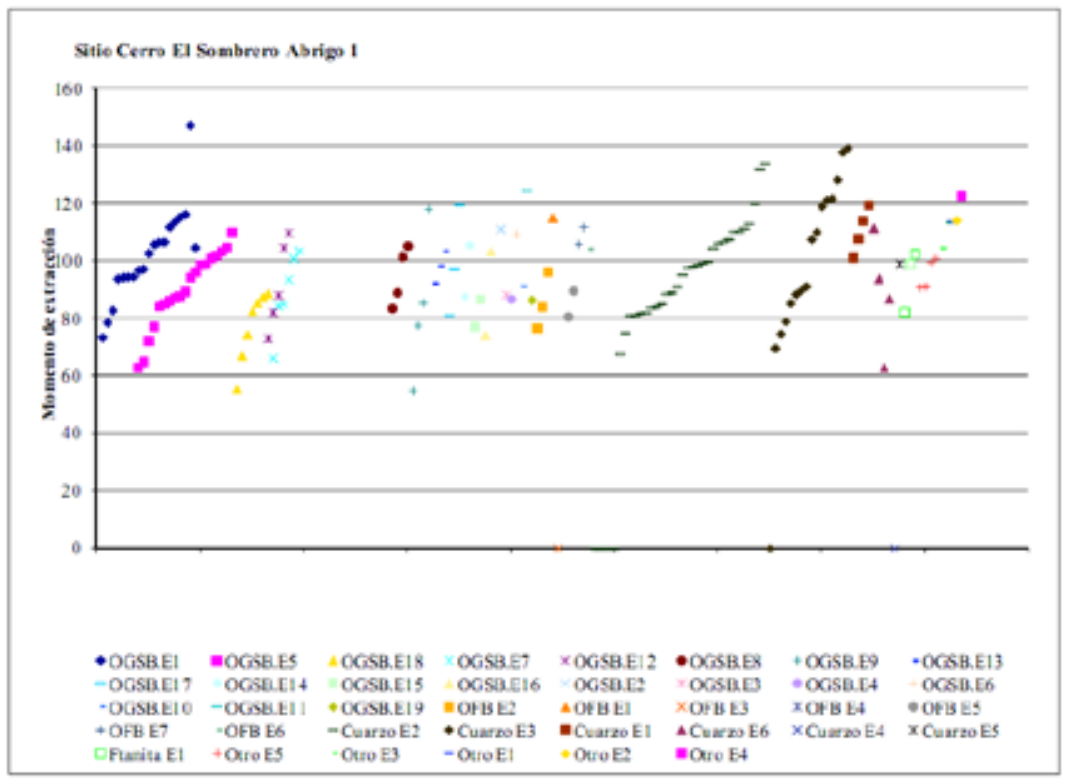

FIG. 7. Resultado del análisis no tipológico obtenido al aplicar el Modelo 4 (Ingbar et al., 1989) para las lascas del sitio Cerro El Sombrero Abrigo 1. Cada conjunto con la misma referencia representa los desechos de talla correspondientes a un mismo nódulo mínimo y cada punto corresponde a un desecho de talla 


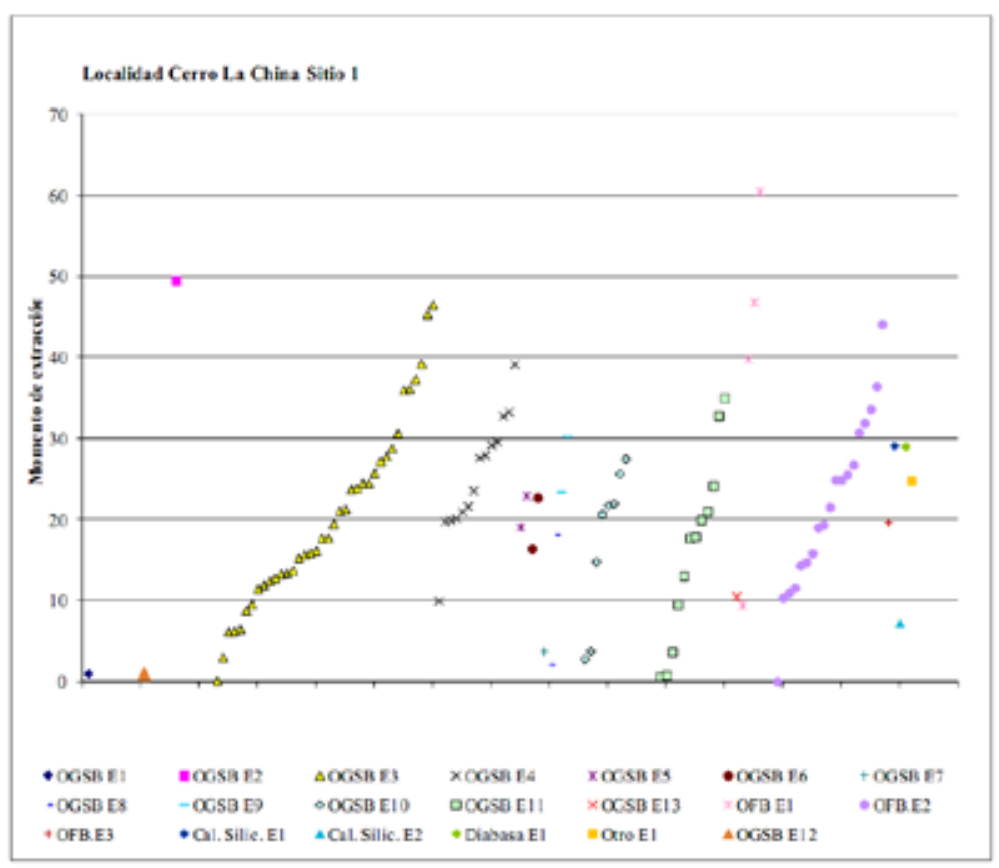

Fig. 8. Resultado del análisis no tipológico obtenido al aplicar el Modelo 4 (Ingbar et al. 1989) para las lascas del sitio Cerro La China 1. Cada conjunto con la misma referencia representa los desechos de talla correspondientes a un mismo nódulo mínimo y cada punto corresponde a un desecho de talla.

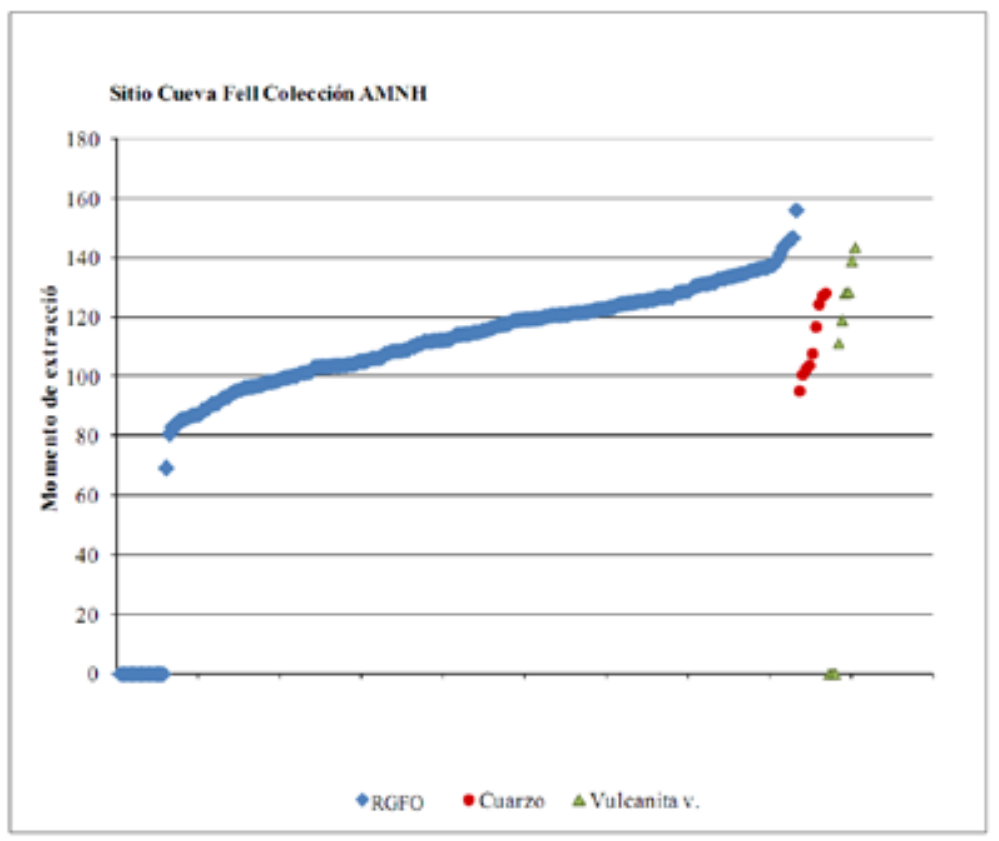

Fig. 9. Resultado del análisis no tipológico obtenido al aplicar el Modelo 4 (Ingbar et al. 1989) para las lascas del sitio Cueva Fell, Colección AMNH.

Cada conjunto con la misma referencia representa los desechos de talla correspondientes a un mismo nódulo mínimo y cada punto corresponde a un desecho de talla. 


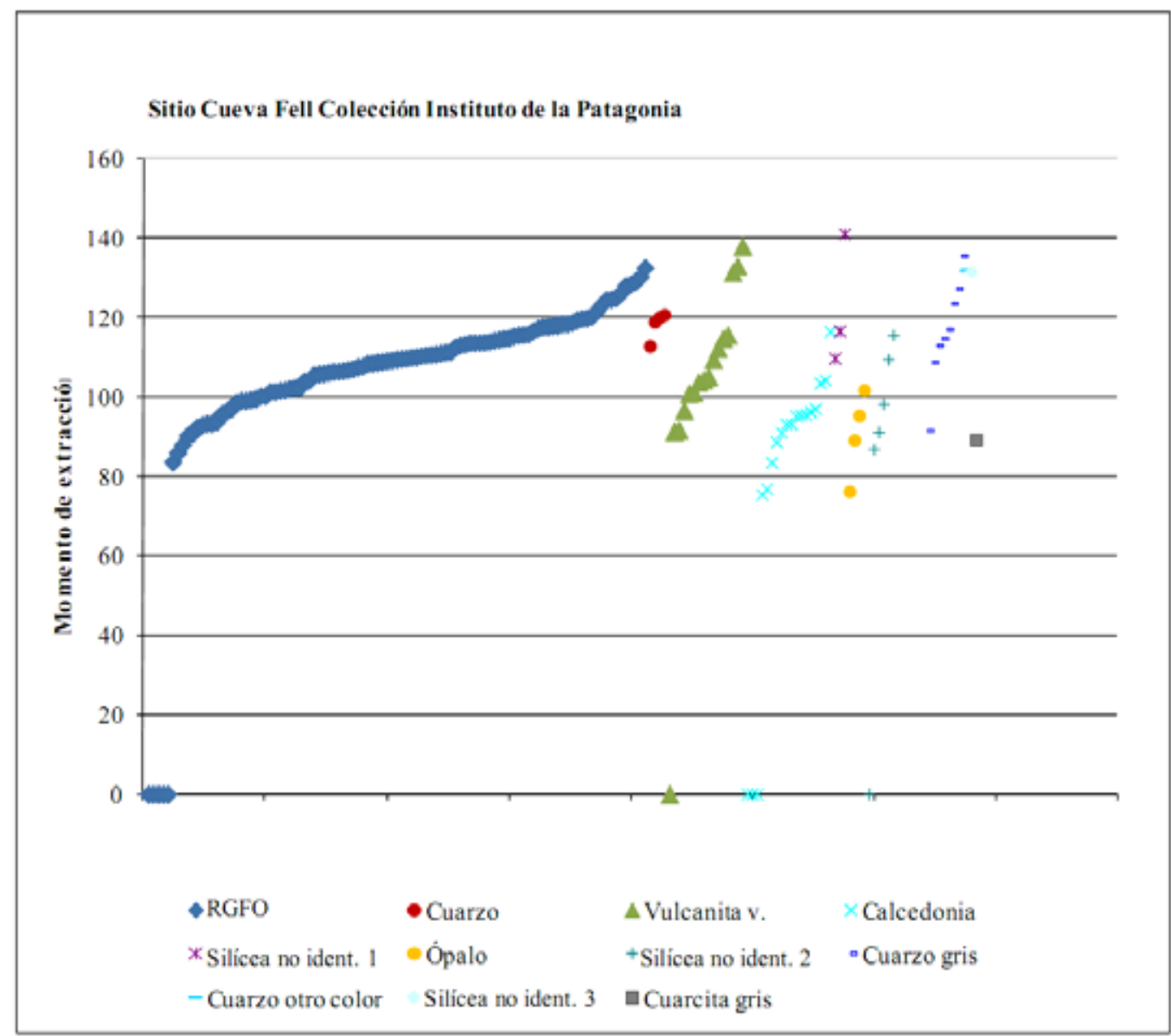

Fig. 10. Resultado del análisis no tipológico obtenido al aplicar el Modelo 4 (Ingbar et al. 1989) para las lascas del sitio Cueva Fell, Colección John Fell (Centro de Estudios del Hombre Austral, Intituto de la Ptagonia, Universidad de Magallanes). Cada conjunto con la misma referencia representa los desechos de talla correspondientes a un mismo nódulo mínimo y cada punto corresponde a un desecho de talla.

Tabla 3. Promedio de momento de extracción para los eventos de talla de la ortocuarcita Grupo Sierras Bayas y el cuarzo del sitio Cerro El Sombrero Abrigo 1.

\begin{tabular}{|c|c|c|}
\hline $\begin{array}{c}\text { Nombre del } \\
\text { evento }\end{array}$ & Promedio & $\begin{array}{c}\text { Desviación } \\
\text { estándar }\end{array}$ \\
\hline OGSB E1 & 68,20 & 13,93 \\
\hline OGSB E5 & 66,08 & 9,91 \\
\hline OGSB E 7 & 67,46 & 7,36 \\
\hline OGSB E 12 & 69,92 & 8,97 \\
\hline OGSB E 18 & 66,96 & 10,85 \\
\hline Cuarzo E2 & 54,19 & 24,45 \\
\hline Cuarzo E3 & 62,98 & 19,13 \\
\hline
\end{tabular}

\section{CERRO LA CHINA SITIO 1}

En un total de 143 lascas se pudieron identificar 20 eventos de talla de distintos nódulos. Con respecto a la ortocuarcita GSB se aislaron al menos 17 eventos de talla diferentes, que reúnen la mayor cantidad del material (Tabla 1). Como ya hemos mencionado, se hace difícil la diferenciación en eventos entre las lascas blancas, sin embargo la curva de regresión que incluye los artefactos blancos se comporta de forma semejante a las curvas que representan los otros nódulos (Fig. 8).

Para el caso de la ortocuarcita FB la secuencia de reducción comienza a estar claramente representada después de la extracción número 10. Los eventos finalizan hacia la extracción 50, sin embargo, existen 
extracciones escasas de momentos posteriores. Para el caso de las ftanitas, calizas silicificadas y otras materias primas sólo está representada una parte muy reducida de la producción de la que formaron parte y esta cae dentro del mismo rango de extracción que las rocas mayoritarias (Fig. 8).

Los promedios de los momentos de extracciones previstas para este sitio son los más bajos de los aquí analizados, varían entre la extracción 32 y la 40 (Tabla 4). El promedio más bajo corresponde a los desechos de ortocuarcita FB.

Tabla 4. Promedio de momento de extracción para los eventos de talla de la ortocuarcita Grupo Sierras Bayas y ortocuarcita Formación Balcarce del sitio Cerro La China 1.

\begin{tabular}{|c|c|c|}
\hline $\begin{array}{c}\text { Nombre del } \\
\text { evento }\end{array}$ & Promedio & $\begin{array}{c}\text { Desviación } \\
\text { estándar }\end{array}$ \\
\hline OGSB E1 & 39,57 & 14,21 \\
\hline OGSB E3 & 40,21 & 12,33 \\
\hline OGSB E 4 & 36,87 & 9,54 \\
\hline OGSB E 10 & 34,97 & 9,07 \\
\hline OGSB E11 & 36,21 & 10,85 \\
\hline OFB E2 & 32.56 & 12.91 \\
\hline
\end{tabular}

\section{SITIO CUEVA FELL}

Si bien las colecciones se consideraron por separado de acuerdo a su proveniencia, las interpretaciones se realizaron en conjunto, ya que como se puede ver en las figuras 9 y 10 ambas colecciones dieron resultados semejantes. Se registró un total de 397 lascas en las que se distinguieron 13 eventos (se unifican los eventos sobre RGFO y vulcanita verde de ambas colecciones) (Tabla 1). Para el caso de los desechos de RGFO, al igual que con los de ortocuarcita blanca pampeana, resulta difícil establecer una separación entre eventos dada la uniformidad de la materia prima. Por ello, aún más que en los casos anteriores, en este sitio los eventos deben ser considerados como un número mínimo. En cuanto a la secuencia de talla en sí, se observa una escasa presencia de lascas iniciales, seguida por una notable ausencia hasta alrededor del momento 70 , donde se registran extracciones con continuidad hasta aproximadamente la número 150 (Fig. 9 y 10). Las rocas minoritarias (vulcanitas, ópalos, calcedonias y cuarzos) presentan curvas de regresión similares a las RGFO, con unas pocas evidencias del inicio del proceso y mayor cantidad de desechos después de la extracción 70.

Los promedios de este conjunto dan valores entre
50 y 78 extracciones (Tablas 5 y 6). Los promedios de RGFO brindan valores de desviación estándar de alrededor de 20 y 25, este valor es alto debido a la presencia de lascas de los primeros momentos de extracción.

Tabla 5. Promedio de momento de extracción para los eventos de talla de Cueva Fell- AMNH.

\begin{tabular}{|c|c|c|}
\hline Nombre del evento & Promedio & $\begin{array}{c}\text { Desviación } \\
\text { estándar }\end{array}$ \\
\hline RGFO & 75,43 & 24,62 \\
\hline Sílice no ident. 1 & 49,19 & 41,34 \\
\hline Cuarzo gris & 77,91 & 10,31 \\
\hline
\end{tabular}

Tabla 6. Promedio de momento de extracción para los eventos de talla de Cueva Fell- Instituto de la Patagonia.

\begin{tabular}{|c|c|c|}
\hline Nombre del evento & Promedio & $\begin{array}{c}\text { Desviación } \\
\text { estándar }\end{array}$ \\
\hline RGFO & 76,40 & 20,01 \\
\hline Vulcanita verde & 70,44 & 21,30 \\
\hline Cuarzo & 73,44 & 5,71 \\
\hline Calcedonia & 59,82 & 29,22 \\
\hline Sílice no ident.2 & 73,47 & 9,91 \\
\hline Ópalo & 68,98 & 12,51 \\
\hline Otra & 57,65 & 34,07 \\
\hline Cuarcita & 66,84 & 35,60 \\
\hline Cuarzo gris & 70,69 & 6,25 \\
\hline
\end{tabular}

\section{DISCUSIÓN}

Los tres sitios pampeanos presentan semejanzas en cuanto a la cantidad de lascas analizadas y eventos identificados (Tabla 1). En cambio, como ya mencionamos, en el caso patagónico aunque el número de lascas es mayor, se reconocieron menos eventos por la dificultad metodológica relacionada a la homogeneidad macroscópica de las materias primas.

En cuanto a la comparación general de los conjuntos de los distintos sitios se observa que las secuencias representan momentos avanzados de manufactura de las piezas, salvo en Cerro La China Sitio 1, donde las curvas finalizan alrededor de la extracción número 50. En Cerro El Sombrero Abrigo 1, Cima y en Cueva Fell la distribución de los desechos en el modelo es semejante: comienzan entre la extracción prevista 50 y 70, y las secuencias finalizan entre el momento 110 o 160 . Asimismo, tanto en Cueva Fell como en Cerro El Sombrero Abrigo 1 se registran algunos escasos eventos de comienzo de la reducción. Esas primeras extracciones posiblemente no correspondan a la producción de 
formas base para tallar puntas de proyectil cola de pescado medianas y grandes ya que corresponden a materias primas en las que no se registraron puntas. En otros casos, las lascas son más pequeñas que las formas base necesarias para la manufactura de los instrumentos de tamaño promedio registrados en los contextos.

Estas apreciaciones son compatibles con las interpretaciones previas de los sitios en el caso pampeano, donde Cerro La China Sitio 1 fue identificado como un lugar de actividades domésticas y variadas donde se realizaron diversas actividades de talla, y Cerro El Sombrero Abrigo 1 y Cima han sido interpretados como lugares donde se realizaron actividades específicas (Flegenheimer 2004; Flegenheimer \& Leipus 2007; Mazzia \& Flegenheimer 2012). Sin embargo, discrepan en cierta medida con las primeras interpretaciones de Cerro El Sombrero Abrigo 1, donde se consideró que varios momentos de la secuencia de reducción estaban representados en el sitio (Flegenheimer 2003). Según el estudio actual, los ocupantes del abrigo habrían descartado pocas lascas de comienzos de la reducción, dado que la mayoría de las extracciones corresponden a momentos avanzados en la manufactura. Algo semejante ocurre en Cueva Fell. Esta cueva, ha sido interpretada como un espacio doméstico donde entre otras actividades, se tallaron puntas; según este estudio de desechos, esta cueva es un lugar donde se desarrollaron con preferencia los momentos finales de manufactura de artefactos con secuencias largas. La indole exploratoria de este trabajo no permite avanzar sobre qué artefactos se estaban elaborando alli. Podría ser un sitio semejante a Cerro El Sombrero Abrigo 1 donde posiblemente se tallaron núcleos y artefactos de ortocuarcita GSB que ya entraron al sitio con muchas extracciones, o un caso como Cerro El Sombrero Cima donde las actividades de talla fueron más específicas y en gran medida debieron estar vinculadas a la manufactura de puntas cola de pescado.

Las diferencias en los modelos también pueden servir para explorar los distintos modos de uso de las materias primas en los casos estudiados (Fig. 6, 7 y 8). En tal sentido, en las rocas mayoritarias (ortocuarcita GSB y RGFO) los eventos que corresponden a la talla de instrumentos de cadenas cortas o a momentos iniciales de secuencias más largas, sólo están presentes en Cerro La China 1, aunque en forma discontinua.
Este es el único sitio de los aquí analizados donde los núcleos habrían sido transportados hacia el sitio con pocas extracciones previas. En cambio, como recién mencionamos, para el caso de las RGFO de Cueva Fell la mayor frecuencia de desechos corresponde a los momentos finales de manufactura (Fig. 9 y 10) y se recuperaron evidencias mínimas de los momentos iniciales. En este último caso, por las características de la materia prima, no podemos evaluar a cuántos eventos correspondieron ni su representatividad. La información bibliográfica de Patagonia nos permite aseverar que las RGFO están disponibles en el área cercana al sitio en un radio menor a 40 km (Nami 1999; Charlin 2007a y b; Jackson 2007; Borrero \& Charlin 2010). Como consideración más general, se observa que es muy notoria la escasez de desechos que corresponden al comienzo de la reducción de núcleos para las rocas mayoritarias en ambas regiones (ver Fig. 6 a 9). Por ello, esta actividad debió realizarse principalmente fuera de los sitios estudiados, incluso posiblemente en las mismas áreas de aprovisionamiento.

Además de estas materias primas mayoritarias, en ambas regiones también se emplearon otras de forma más esporádica. En Cueva Fell las vulcanitas verdes y las calcedonias siguen en importancia a las RGFO. En Pampa, la ortocuarcita FB es escasa en ambos sitios de Cerro El Sombrero y más frecuente en Cerro La China Sitio1, los cuarzos, en cambio, son más frecuentes en Cerro El Sombrero Abrigo 1, y en Cima casi no se emplearon otras rocas además de ortocuarcita GSB. En los sitios pampeanos, la ortocuarcita FB y los cuarzos están inmediatamente disponibles y esta distribución en el paisaje ha sido relevante para explicar cómo fueron empleados (Bayón et al. 2006). Por ejemplo, en Cerro El Sombrero Abrigo 1 (Fig. 7) se puede ver que hubo una diferencia en el tratamiento entre estas rocas inmediatas y las que provienen de canteras más distantes, como la ortocuarcita GSB. Asimismo, estos cuarzos disponibles en el cerro probablemente no fueron empleados en la manufactura de puntas de proyectil cola de pescado sino en artefactos con otras secuencias de talla. Cabe destacar que las puntas cola de pescado de cuarzo halladas en Cima están talladas sobre otras variedades de esta roca que no se ha observado en las inmediaciones de los sitios. Asimismo, la discontinuidad de las curvas de estas rocas en Cerro La China Sitio 1 (Fig 8) y en Cerro 
El Sombrero Abrigo 1 (Fig 7) podría deberse a que algunas lascas de mejor calidad hayan sido empleadas como formas base de artefactos formatizados. Algunos de estos instrumentos han sido descriptos en los sitios y para el caso de la ortocuarcita FB también se conoce una preforma de punta cola de pescado (Flegenheimer 1986-87).

Finalmente, otras materias primas con una escasa representación en los conjuntos pampeanos son la calizas silicificadas y las ftanitas; en Patagonia son cuarzos, cuarcitas y otras rocas silíceas no identificadas que se encuentran en forma de clastos nodulares. Es interesante notar que estas rocas, de las que hay pocos desechos y que representan sólo una parte de la secuencia de reducción, se trataron del mismo modo que las materias primas mayoritarias en cada sitio. Es decir, la representación de los desechos no responde a una trayectoria esperable de la roca en relación a su calidad, disponibilidad y proveniencia. Esto incluso puede argumentarse en el caso de las calizas silicificadas que provienen de larga distancia en la región pampeana y que posiblemente circularon como artefactos ya formatizados dentro de redes sociales amplias. Una de las evidencias que sostienen este planteo es la existencia de escasas lascas que corresponden a las últimas etapas de formatización (Flegenheimer et al. 2003). En el trabajo actual puede verse que estas lascas, aunque muy escasas, siguen la misma tendencia que los desechos restantes en los sitios, es decir representan momentos de extracción más tempranos en Cerro La China Sitio 1 que en Cerro El Sombrero (ver Figs. 6 a 8).

En cuanto a la metodología aplicada, ésta ha resultado una herramienta útil y ágil para ordenar los desechos analizados. En el caso pampeano es interesante observar las diferencias notorias entre los desechos de Cerro La China 1 y los sitios de Cerro El Sombrero. Aunque la misma era esperable a partir de las interpretaciones previas de los sitios y de la tipología de desechos tradicional, los gráficos son muy contundentes al respecto (Fig. 6, 7 y 8). Lo mismo ocurre en cuanto a la homogeneidad de los desechos de Cerro El Sombrero Cima; aunque este tema ya se mencionó a partir de estudios tecno-morfológicos previos (Zárate et al. 2000-2002, Flegenheimer \& Mazzia 2013), su representación gráfica en este trabajo resulta muy clara. Como la metodología se restringió aquí al análisis de los desechos, no se exploró todo el potencial interpretativo del método. Sin embargo, consideramos que esta aplicación exploratoria del método ha resultado útil a los fines comparativos y sería interesante incorporar los artefactos restantes a estudios posteriores.

\section{CONCLUSIONES}

Luego de este análisis comparativo podemos hacer algunas observaciones generales. En primera instancia, resalta la discontinuidad de las trayectorias en todos los conjuntos, prácticamente sólo un segmento de la secuencia de manufactura está representado en cada sitio. También es notoria la semejanza en las trayectorias representadas en Cueva Fell, Cerro El Sombrero Abrigo 1 y en cierta medida, Cima. En cambio, las trayectorias de Cerro La China Sitio 1 terminan aproximadamente donde comienzan las restantes, es decir, no hay superposición en los trayectos realizados entre este sitio y los demás.

Al considerar las trayectorias en más detalle, de acuerdo a los momentos de talla observamos que los momentos finales de manufactura son los más representados. En el sitio Cima el rango es el más restringido lo cual indica que en este lugar probablemente las actividades de talla fueron más específicas. Es decir, esta trayectoria más acotada en Cima, coincide con su especificidad como lugar especial en el paisaje, tema que viene siendo remarcado desde el comienzo de las investigaciones y sostenido desde distintos estudios (Flegenheimer 2003, Flegenheimer \& Mazzia 2013).

En cuanto a nuestros objetivos originales, la aplicación de esta metodología a desechos ya trabajados produjo en gran medida, resultados comparables con los obtenidos anteriormente sobre los momentos de las secuencias de manufactura representados en los distintos sitios y materias primas. También consideramos que estos resultados refuerzan la propuesta que se viene sosteniendo para Pampa acerca de una vinculación muy estrecha entre los espacios y las prácticas que en ellos desarrollaron sus ocupantes del Pleistoceno tardío y Holoceno temprano. Asimismo deja abierta la posibilidad de que esta modalidad de asignación de los espacios a ciertos usos, se aplique en ambas regiones y sea parte del modo de hacer de los ocupantes tempranos.

Finalmente, la discontinuidad en las tareas de talla nos hace reflexionar acerca de estos lugares y su gente. Si se trató de espacios en los que desarrollaron actividades específicas, ¿quiere decir que fueron ocupados por algún sector particular de la comunidad? ¿Cuál en 
cada caso? Como dijimos, los resultados parecen indicar que la toma de decisiones en torno a la producción lítica estuvo orientada a identificar ciertos espacios en los que la gente vivió o trabajó, con determinadas actividades de talla. En tal caso, resulta interesante que esta modalidad sea compartida en regiones distantes por gente que sabemos que también compartió los conocimientos necesarios para fabricar instrumentos como las puntas cola de pescado y las piedras discoidales. Por ello, concordamos con quienes proponen que posiblemente estemos viendo evidencias de intercambio de información tecnológica a gran escala, entre grupos humanos distintos (Massone \& Prieto 2004). Es decir, a partir de este trabajo proponemos que además de los conocimientos prácticos de cómo realizar una tarea, posiblemente también se estuvieran compartiendo otras cuestiones más relacionadas a la esfera de los vínculos entre la gente y su entorno, o sea cuestiones que conciernen a la compleja trama de decisiones que implica la tecnología entendida en su sentido amplio.

\section{AGRADECIMIENTOS}

Este trabajo ha sido posible gracias a un subsidio de la Wenner Gren Foundation, a un Collection Grant del American Museum of Natural History (R.C.) y a subsidios PIP 112-200801-02979 y PICT 2010 No.1517 (N. F) y PIP 112-200901-00191 y PICT 2011 No. 2122 (RC). Las autoras desean agradecer especialmente a la curadora de las colecciones del AMNH, Sumru Aricanli. Asimismo el estudio de la colección que se encuentra en Punta Arenas fue posible gracias al apoyo del Instituto de la Patagonia, su director, Mateo Martinic, y nuestros colegas Flavia Morello, Gabriel Bahamonde y Pedro Cárdenas. Andres Izeta, R. Barberena, Natalia Mazzia, Cristina Bayón, Celeste Weitzel y Vale Lukezic colaboraron con fotos, imágenes y bibliografía. Agradecemos los consejos de los revisores del manuscrito original que ayudaron a mejorar la calidad del texto.

\section{BIBLIOGRAFÍA}

Ahler, S. A. (1989). Mass Analysis of Flaking Debris: Studying the Forest Rather Than the Trees. Alternative Approaches to Lithic Analysis. Archaeological Papers of the American Anthropological Association 1, 85-118.

Andrefsky, W. (2005). Lithic Studies. En H.D.G. Maschner \& C. Chippindale (Eds.), Handbook of Methods in Ar- chaeology, (pp. 713-770). Walnut Creek: AltaMira Press. Aschero, C. (1975). Ensayo para una clasificación morfológica de artefactos líticos aplicada a estudios tipológicos comparativos. Informe CONICET. Buenos Aires.

Aschero, C. (1983). Ensayo para una Clasificación Morfológica de Artefactos. Apéndice A y B. Cátedra de Ergología y Tecnología, Facultad de Filosofía y Letras, Universidad de Buenos Aires. Buenos Aires.

Aschero, C. A. (1988). De punta a punta: producción, mantenimiento y diseño de puntas de proyectil precerámicas de la puna argentina. Ponencia presentada en IX Congreso Nacional de Arqueología Argentina. Simposio: Las Unidades de Análisis para el estudio del cambio cultural en Arqueología (pp.229). Buenos Aires

Ashton, N. M \& M. J. White (2003). Bifaces and Raw materials: flexible flaking in the British Lower Palaeolithic.. En Dibble, H. \& Sorresi (Eds.), Multiple Approaches to the study of Bifacial Technologies (109-124). Pennsylvania: University of Pennsylvania Museum Press.

Audouze F. (1999). New advances in French Prehistory. Antiquity 73, 167-175.

Bamforth, D. A \& N. Finlay (2008). Introduction: Archaeological Approaches to lithic production skill and craft learning. Journal of Archaeological Method and Theory.15, 1-27.

Bayón, C., N. Flegenheimer \& A. Pupio (2006). Planes Sociales en el Abastecimiento y Traslado de Roca en la Pampa Bonaerense en el Holoceno Temprano y Tardío. Relaciones de la Sociedad Argentina de Antropología XXXI, 19-45.

Bellelli, C., A. G. Guráieb \& J. A. García (1985-87). Propuesta para el análisis y procesamiento por computadora de desechos de talla lítica (DELCO-Desechos líticos computarizados). Arqueología Contemporánea 2(1), 36-53

Bird, J. (1938). Antiquity and migrations of the early inhabitants of Patagonia. The Geographical Review 28, 250-275.

Bird, J. (1988). Travels and Archaeology in South Chile. Iowa: University of lowa Press.

Boeda, P. (1996). Les Chasseurs Magdaleniens de Pincevent; quelques aspects de leurs Comportements. Lithic Technology 21 (1), 48-70.

Bradbury, A. P. \& J. D. Franklin (2000). Raw Material Variability, Package Size and Mass Analysis. Lithic Technology, 25(1), 62-78.

Borrero, L. \& J. Charlin (2010). Arqueología de Pali Aike y Cabo Vírgenes. Buenos Aires: Editorial Dunken.

Castiñeira, C., M. Cardillo, J. Charlin, y J. Baeza. (2011) Análisis de morfometría geométrica en puntas cola de pescado del Uruguay. Latin American Antiquity 22(3), 335-58. 
Cattáneo, G. R. (2002). Una Aproximación a la Organización de la Tecnología Lítica entre los Cazadores- Recolectores del Holoceno Medio/Pleistoceno Final en la Patagonia Austral, Argentina. Doctorado, Universidad Nacional de La Plata, La Plata.

Cattáneo, G. R. (2006). Tecnología Lítica del Pleistoceno Final/Holoceno Medio Un Estudio de los CazadoresRecolectores de la Patagonia Austral (Argentina). Oxford: Archaeopress (BAR S1580).

Charlin, J. (2005). Aprovisionamiento de materias primas líticas en el campo volcánico de Pali Aike (Santa Cruz): una primera aproximación a partir del análisis de los núcleos. Werken 7, 39-55.

Charlin, J. (2006). Los instrumentos líticos en Pali Aike (Pcia. Santa Cruz): ¿Homogeneidad o diversidad en la utilización de las materias primas líticas? Werken 9, 47-71.

Charlin, J. (2007a). Una perspectiva espacial de la intensidad de uso de las materias primas líticas en el campo volcánico Pali Aike (Prov. Santa Cruz, Argentina). En F. Morello, M. Martinic, A. Prieto y G. Bahamonde (Eds.), Arqueología de Fuego-Patagonia. Levantando piedras, desenterrando huesos... y develando arcanos., (pp. 129-138). Editorial CEQUA, Punta Arenas.

Charlin, J. (2007b). Explorando la intensidad de uso de las materias primas líticas en Pali Aike (Prov. Santa Cruz, Argentina). Intersecciones en Antropología 8, 287-299.

Charlin, J. (2009). Estrategias de aprovisionamiento y utilización de las materias primas líticas en el campo volcánico Pali Aike (Prov. de Santa Cruz, Argentina). Oxford: Archaeopress (BAR S1901).

Colombo, M. \& N. Flegenheimer (2011). La elección de rocas de colores por los pobladores tempranos de las sierras de Lobería (Buenos Aires, Argentina). Nuevas consideraciones desde las canteras. Enviado para su publicación.

Dibble, H. (1997). Platform variability and flake morphology: a comparison of experimental and archaeological data and implications for interpreting prehistoric lithic technological strategies. Lithic Technology 22(2), 150-170.

Dibble, H. \& M. Bernard (1980). A comparative study of basic edge angle measurement techniques. American Antiquity 45, 857-865.

Emperaire , J., A. Laming-Emperaire, H. Reichlen \& T. PoulainJosien (1963). La grotte Fell et autres sites de la région volcanique de la Patagonie chilienne. Journal de la Société des Amèricanistes 52, 167-254.

Espinosa, S. (1998). Desechos de talla: tecnología y uso del espacio en el Parque Nacional Perito Moreno (Santa Cruz, Argentina). Anales del Instituto de la Patagonia. Serie Ciencias Humanas 26, 153-168.
Flegenheimer, N. (1986-87). Excavaciones en el Sitio 3 de la Localidad Cerro La China (Prov. de Bs.As.). Relaciones de la Sociedad Argentina de Antropología XVII(I), 7-28.

Flegenheimer, N. (1988). Producción lítica en el Cerro El Sombrero, Resultados preliminares. En Libro de Resúmenes, IX Congreso Nacional de Arqueología Argentina (pp. 58), Buenos Aires.

Flegenheimer, N. (1991). Bifacialidad y Piedra con picado, Abrasión y Pulido en Sitios Pampeanos Tempranos. Shincal 3(2), 64-78.

Flegenheimer, N. (1999). ¿Vista una, vistas todas? Las "Colas de Pescado" de la Cima del Cerro El Sombrero. En Resúmenes del XIII Congreso Nacional de Arqueología Argentina (pp. 353-354), Córdoba.

Flegenheimer, N. (2003). Cerro El Sombrero, a locality with a view. En L. Miotti, M. Salemme \& N. Flegenheimer (Eds.), Where the South Winds Blow, Ancient Evidence of Paleo South Americans (pp.51-56). Texas: A \& M University Press, Center for the Study of the First Americans.

Flegenheimer, N. (2004). Las Ocupaciones de la transición Pleistoceno-Holoceno: una visión sobre las investigaciones en los últimos 20 años en la Región pampeana. En L. Beovide, I. Barreto \& C. Curbelo (Eds.), Actas del X Congreso Nacional de Arqueología Uruguaya. Publicación en CD.

Flegenheimer, N. \& M. Zárate (1997). Considerations on radiocarbon and calibrated dates from Cerro La China and Cerro El Sombrero, Argentina. Current Research in the Pleistocene 14, 27-28.

Flegenheimer, N. \& C. Bayón (1999). Abastecimiento de rocas en sitios pampeanos tempranos: recolectando colores. En Aschero, C., M. A. Korstanje \& P. M. Vuoto (Eds.), En los Tres Reinos: Prácticas de recolección en el Cono sur de América. (pp. 95-111). Tucumán: Ediciones Magna Publicaciones, Instituto de Arqueología.

Flegenheimer, N., C. Bayón, M. Valente, J. Baeza \& J. Femenías (2003). Long Distance Tool Stone Transport in the Argentine Pampas. L. Miotti y M. Salemme (Eds.), Quaternary International, 109-110, 49-64.

Flegenheimer, N. \& M. Leipus (2007). Trabajar en un espacio reducido, Cerro El Sombrero, Abrigo 1. Actas del XVI Congreso Nacional de Arqueología Argentina (pp.441444), Córdoba.

Flegenheimer, N \& N. Mazzia (2013). Cerro El Sombrero Cima, un lugar particular para los pobladores tempranos. Revista del Museo de La Plata. En prensa.

Frison, G. (1974). The Casper Site. A Hell Gap Bison Kill on the High Plains. New York: Academic Press.

Gnecco, C. (2000). Ocupación temprana de bosques tropicales 
de montaña. Popayán: Universidad del Cauca.

Ingbar, E., M. Larson \& B. Bradley (1989). A non typological approach to débitage analysis. En D. Amick \& R. Mauldin (Eds.). Experiments in lithic technology. (pp. 67-99). Oxford: Archaeopress (BAR S528).

Jackson, D. (2007). Estructura, Intensidad y reiteración en las ocupaciones paleoindias en cuevas y aleros de Patagonia Meridional, Chile. Revista de Arqueología de cazadoresrecolectores del Cono Sur 2, 67-87.

Jackson S. D. \& A. Prieto (2005). Estrategias tecnológicas y conjunto lítico del contexto paleoindio de Cueva Lago Sofía 1, Última Esperanza, Magallanes. Magallania, 33(1), 115-120.

Jackson, D. \& C. Méndez (2007). Litos discoidales tempranos en contextos de Patagonia. Magallania 35(1), 43-52.

Kelly, R. L. (1985). Hunter-Gatherer Mobility and Sedentism: A Great Basin Study. Doctorado, University of Michigan.

Kuijt, I \& K.W. Rusell (1993). Tur Imdai Rockshelter, Jordan: Debitage analysis and historic Bedouin lithic technology. Journal of Archaeological Science 20, 667-680.

Laming-Emperaire, A. (1967). Cadre cronologique provisoire de la prehistoire de Patagonie et de Terre de Feu Chiliennes. Boletín del Museo Nacional de Historia Natural 30, 221-236.

Larson, M. (1994). Toward a holistic analysis of chipped stone assemblages. En P. Carr (Ed), The organization of North American Prehistoric Chipped Stone Technologies. Michigan: International Monographs in Prehistory Archaeological Series 7.

Larson M. L. \& M. Kornfeld (1997). Chipped stone nodules: theory, method, and examples. Lithic Technology $22,4-18$.

Martínez, G. (2006). Arqueología del curso medio del río Quequén Grande: estado actual y aportes a la arqueología de la Región Pampeana. Relaciones de la Sociedad Argentina de Antropología XXXI, 249-275.

Martínez, G., C. Landini \& M. Bonomo (1997-1998). Análisis de los artefactos líticos del sitio Paso Otero 3: Una aproximación al entendimiento de la organización de la tecnología lítica en el curso medio del Río Quequén Grande. En Publicaciones de Arqueología, 49. Córdoba: Centro de Investigaciones de la Facultad de Filosofía y Humanidades de la Universidad Nacional de Córdoba.

Massone, M. (1981). Arqueología de la región volcánica de Pali Aike (Patagonia Meridional chilena). Anales del Instituto de la Patagonia 12, 95-124.

Massone, M. (1996). Hombre temprano y paleoambiente en la región de Magallanes: evaluación crítica y perspectivas. Anales del Instituto de la Patagonia 24, 81-98.
Massone M. \& A. Prieto (2004). Evaluación de la modalidad cultural Fell 1 en Magallanes. Chungara, Revista de Antropología Chilena Volumen Especial, 303-315.

Mayer Oakes, W. (1973). Obsidian tell-tales useful in studies of stone flaking techniques. En Lathrap D.W. \& Douglas, J. (Eds.), Variation in Anthropology, (pp. 55-59). Urbana: Illinois Archaeological Survey.

Mazzanti, D. (2003). The Pleistocene-Holocene stratigraphic record from Early Archaeological Sites in caves and Rockshelters of Eastern Tandilia, Pampean Region, Argentina. En Miotti, L., Salemme, M \& Flegenheimer, N. (Eds.), Where the winds blow, Ancient evidence of Paleo South Americans. (pp. 57-61). Texas: A\&M University Press.

Mazzia, N. (2010/2011). Lugares y paisajes de cazadoresrecolectores en la pampa bonaerense: cambios $y$ continuidades durante el Pleistoceno final-Holoceno. Doctorado, Universidad Nacional de La Plata.

Mazzia, N. \& N. Flegenheimer (2007). Retorno a La China: una visión de las ocupaciones tardías. En Bayón, M., Pupio,N., González, M., Flegenheimer, N. \& Frere (Eds.), Arqueología en las pampa (pp. 549-565). Buenos Aires: Sociedad Argentina de Antropología.

Mazzia N. \& N. Flegenheimer (2012). Early settlers and their places in the Tandilia range (Pampean region, Argentina). En Miotti, L., Salemme, M., Flegenheimer, N. \& Goebel, T. (Eds.), Southbound, Late Pleistocene Peopling of Latin America, Special Edition Current Research in the Pleistocene (pp. 105-110). Texas: A\&M University Press.

Nami, H. (1991). Desechos de talla y teoría de alcance medio: un caso de península Mitre, Tierra del Fuego. Shincal 3, 94-112.

Nami, H. (1993-94). Observaciones sobre desechos de talla procedentes de las ocupaciones tempranas de Tres Arroyos (Tierra del Fuego, Chile). Anales del Instituto de la Patagonia. 22, 175-180.

Nami, H. (1997). Investigaciones actualísticas para discutir aspectos técnicos de los cazadores-recolectores del Tardi-glacial: el problema Clovis-Cueva Fell. Anales del Instituto de la Patagonia 25, 152-186.

Nami, H. (1999). Arqueología en la localidad arqueológica de Pali Aike, cuenca del río Chico (Provincia de Santa Cruz, Argentina). Praehistoria 3, 189-218.

Nami, H. (2003). Experimentos para explorar la secuencia de reducción Fell de la Patagonia Austral. Magallania 31, 107-138.

Orquera L. \& E. Piana (1987) Normas para la descripción de objetos arqueológicos de piedra tallada. CADIC- 
CONICET, Buenos Aires.

Pautassi, E. \& G. Sario (2011). Talla de extracción, formatización de filo y adelgazamiento bifacial: una aproximación experimental para el estudio de los conjuntos líticos de cuarzo. Presentado en las IX Jornadas de Arqueología de Centro Oeste del País Río Cuarto, Córdoba.

Politis, G. (1991). Fishtail projectile points in the Southern Cone of South America: an overview. En Bonnichsen, R. \& Turnmire K. (Eds.), Clovis. Origins and Adaptations, (pp. 287-301). Orono: Center for the Study of the First Americans.

Politis, G. (2008). The Pampas and Campos of South America. En Silverman, H. \& Isbell, W. (Eds.), Handbook of South American Archaeology (pp. 235-260). New York: Springer.

Prieto, A. (1997). Algunos resultados de los trabajos arqueológicos en Juni Aike 2. Anales del Instituto de la Patagonia 19, 137-146.

Prentiss, W. (1998). The reliability and validity of a lithic debitage tipology: implications for archaeological interpretations. American Antiquity 63(4), 635-650.

Rozen, K. \& A. Sullivan III (1989). Measurement method and meaning in lithic analyisis: Problems with Amick and Mauldin`s Middle Range Approach. American Antiquity 54(1), 169-175.

Sario, G. (2007). Análisis morfo-tecnológico de los materiales líticos del sitio Estancia La Suiza 3 (provincia de San Luis). Resúmenes del XVI Congreso Nacional de Arqueología Argentina. Revista Pacarina III, 497-500.

Sario, G. (2009). Estancia La Suiza 3 (provincia de San Luis): un estudio de la tecnología lítica. La Zaranda de Ideas, Revista de Jóvenes Investigadores en Arqueología 5, 45-64.

Sario, G. \& E. Pautassi (2010). El aprovisionamiento de las rocas. Un caso de estudio en la localidad arqueológica de Estancia La Suiza. En Actas del XVII Congreso
Nacional de Arqueología Argentina. Tomo V, 17411745. Mendoza.

Sario, G. \& E. Pautassi. 2012. Estudio de secuencias de talla lítica a través de modelos experimentales en rocas silíceas del centro de Argentina. Arqueología Iberoamericana 15, 3-12. http://www.laiesken.net/arqueologia/ archivo/2012/15/1.html.

Schidlowsky, V. (2001). Les premiers chasseurs maritimes et les chasseurs terrestres de Patagonie australe Comportements techno-économiques et identité culturelle: contribution de la technologie lithique. Oxford: Archaeopress (BAR S954, Paris Monographs in American Archaeology 10).

Shott, M. J. (1994). Size and form in the analysis of flakes debris: review and recent approaches. Schiffer (Ed). Journal of Archaeological Method and Theory 1, 69-110.

Shott, M. (1996). Stage versus continuum in the debris assemblages from production of a fluted biface. Lithic Technology 21(1), 6-22.

Sullivan, A. \& K. Rozen (1985). Debitage analysis and archaeological interpretation. American Antiquity 50, 755-779.

Teltser, P. (1991). Generalized core technology and tool use: a Mississippian example. Journal of Field Archaeology $18,363-375$.

Weitzel, C. (2010) El estudio de los artefactos formatizados fracturados. Contribución a la comprensión del registro arqueológico y la actividad humana. Doctorado, Universidad de Buenos Aires.

Weitzel C. \& N. Flegenheimer (2007). Cerro El Sombrero: fractured stones on a flat hilltop. Current Research in the Pleistocene 24, 68-70.

Zárate, M.A., M.I. González, De Bonaveri, N. Flegenheimer \& C. Bayón (2000-2002). Sitios arqueológicos someros: El concepto de sitio en estratigrafía y sitio de superficie. Cuadernos del Instituto de Antropología y Pensamiento Latinoamericano 19, 635-653. 